NBER WORKING PAPER SERIES

\title{
INFORMATIONAL CONTENT OF FACTOR STRUCTURES IN SIMULTANEOUS BINARY RESPONSE MODELS
}

\author{
Shakeeb Khan \\ Arnaud Maurel \\ Yichong Zhang \\ Working Paper 28327 \\ http://www.nber.org/papers/w28327 \\ NATIONAL BUREAU OF ECONOMIC RESEARCH \\ 1050 Massachusetts Avenue \\ Cambridge, MA 02138 \\ January 2021
}

We thank Serena Ng, Arthur Lewbel, two anonymous referees, and seminar participants at Arizona State University, Emory, Michigan State, Shanghai University of Finance and Economics, University of Arizona, as well as conference participants at the 2015 SEA meetings for helpful comments. We also thank Zhangchi Ma and Qingsong Yao for excellent research assistance. Zhang acknowledges the fińnancial support from Singapore Ministry of Education Tier 2 grant under grant MOE2018-T2-2-169 and the Lee Kong Chian fellowship The views expressed herein are those of the authors and do not necessarily reflect the views of the National Bureau of Economic Research.

NBER working papers are circulated for discussion and comment purposes. They have not been peer-reviewed or been subject to the review by the NBER Board of Directors that accompanies official NBER publications.

(C) 2021 by Shakeeb Khan, Arnaud Maurel, and Yichong Zhang. All rights reserved. Short sections of text, not to exceed two paragraphs, may be quoted without explicit permission provided that full credit, including $(\odot$ notice, is given to the source. 
Informational Content of Factor Structures in Simultaneous Binary Response Models Shakeeb Khan, Arnaud Maurel, and Yichong Zhang

NBER Working Paper No. 28327

January 2021

JEL No. C14,C21,C25,C38

\begin{abstract}
$\underline{\text { ABSTRACT }}$
We study the informational content of factor structures in discrete triangular systems. Factor structures have been employed in a variety of settings in cross sectional and panel data models, and in this paper we formally quantify their identifying power in a bivariate system often employed in the treatment effects literature. Our main findings are that imposing a factor structure yields point identification of parameters of interest, such as the coefficient associated with the endogenous regressor in the outcome equation, under weaker assumptions than usually required in these models. In particular, we show that a non-standard exclusion restriction that requires an explanatory variable in the outcome equation to be excluded from the treatment equation is no longer necessary for identification, even in cases where all of the regressors from the outcome equation are discrete. We also establish identification of the coefficient of the endogenous regressor in models with more general factor structures, in situations where one has access to at least two continuous measurements of the common factor.

Shakeeb Khan

Department of Economics

Boston College

Chestnut Hill, MA 02467

shakeeb.khan@bc.edu

Arnaud Maurel

Department of Economics

Duke University

213 Social Sciences Building

Box 90097

Durham, NC 27708

and IZA

and also NBER

apm16@duke.edu

Yichong Zhang

School of Economics

Singapore Management University

90 Stamford Rd.

Singapore 178903

Singapore

yczhang@smu.edu.sg
\end{abstract}




\section{Introduction}

Factor models see widespread and increasing use in various areas of econometrics. This type of structure has been employed in a variety of settings in cross sectional, panel and time series models, and have proven to be a flexible way to model the behavior of and relationship between unobserved components of econometric models. The basic idea behind factor models is to assume that the dependence across the unobservables is generated by a low-dimensional set of mutually independent random factors. The applied and theoretical research employing factor structures in econometrics is extensive. In particular, these models are often used in the treatment effect literature as a way to identify the joint distribution of potential outcomes from the marginal distributions, and then recover the distribution of treatment effects from this joint distribution. ${ }^{1}$ Factor models have been used in a number of different contexts in applied microeconomics. These include, among others, earnings dynamics (Abowd and Card, 1989; Bonhomme and Robin, 2010), estimation of returns to schooling and work experiences (Ashworth, Hotz, Maurel, and Ransom, 2020), as well as cognitive and non-cognitive skill production technology (Cunha, Heckman, and Schennach, 2010). Heckman and Vytlacil (2007a,b) provide various additional references. All of these papers, with the notable exception of Cunha, Heckman, and Schennach (2010), rely on linear factor models where the unobservables are assumed to be written as the sum of a linear combination of mutually independent factors and an idiosyncratic shock.

In this paper we bring together the literature on factor models with the literature on the identification and estimation of triangular binary choice models (Chesher (2005); Vytlacil and Yildiz (2007); Shaikh and Vytlacil (2011); Han and Vytlacil (2017)) by exploring the informational content of factor structures in this class of models. ${ }^{2}$ Focusing on this class can be well motivated from both an empirical and theoretical perspective. From the former, many treatment effect models fit into this framework as treatment is typically a binary and endogenous variable in the system, whose effect on outcomes is often a parameter the econometrician wishes to conduct inference on. From a theoretical perspective, inference on this type of system can be complicated, if not impossible without strong parametric assumptions, which may not be reflected in the observed data. Imposing no restriction on the structure of endogeneity often fails to achieve identification of parameter, or at best only do so in sparse regions of the data, thus making inference impractical in practice. In this context, modeling the endogeneity between the selection and the outcome by a factor structure may be a useful "in-between" setting, which, at the very least, can be used to gauge the sensitivity of the parametric approach to their stringent assumptions.

We start our analysis by imposing a particular factor structure to the two unobservables in this system and explore the informational content of this assumption. We assume that the unobservables

\footnotetext{
${ }^{1}$ See also Abbring and Heckman (2007) for an extensive discussion of factor structures and prior studies using these models in the context of treatment effect estimation.

${ }^{2}$ See also recent work by Lewbel, Schennach, and Zhang (2020), who study the identification of a triangular linear model assuming that the disturbances are related through a factor model.
} 
from the treatment equation $(V)$ and the outcome equation $(U)$ are related through the following factor model:

$$
U=\gamma_{0} V+\Pi
$$

where $\Pi$ is an unobserved random variable assumed to be distributed independently of $V$ and $\gamma_{0}$ is a scalar parameter. This structure generalizes the canonical case where the unobservables $(U, V)$ are jointly normally distributed, for which this relationship always holds. Our main finding is that there is indeed informational content of factor structures in the sense that, in contrast to prior literature notably Vytlacil and Yildiz (2007) - one no longer requires an additional "non-standard" exclusion restriction, nor the strong support conditions on the covariates entering the outcome equation that are generally needed for identification in these models. Our identification results are constructive and translate directly into a rank based estimator of the coefficient associated with the binary endogenous variable, which we provide and study in a supplement to this paper.

While an appealing feature of the structure considered in Equation (1.1) is that it is a natural extension of the bivariate Probit specification that has often been considered in the literature, this model does impose significant restrictions on the nature of the dependence between the unobservables $U$ and $V$. In the paper we extend this baseline specification by considering a linear factor structure of the form:

$$
\begin{aligned}
U & =\gamma_{0} W+\eta_{1} \\
V & =W+\eta_{2}
\end{aligned}
$$

where $\left(W, \eta_{1}, \eta_{2}\right)$ are mutually independent unobserved random variables. We study the informational content of this extended factor structure in the context of triangular binary choice models and establish identification, assuming access to at least two continuous noisy measurements of the unobserved factor $W$. This setup has been used in a number of applications, in particular in labor economics. In these applications, the unobserved factor is typically interpreted as latent individual ability, about which several continuous noisy measurements are available from the data. This is the case of, for instance, Carneiro, Hansen, and Heckman (2003), Cunha, Heckman, and Schennach (2010), Heckman, Humphries, and Veramendi (2018) and Ashworth, Hotz, Maurel, and Ransom (2020), who use components of the Armed Services Vocational Aptitude Battery test as measurements of cognitive ability. At a high level, it is interesting to note that these results complement Bai and $\mathrm{Ng}$ (2010), who show that, in the context of a linear regression model with endogenous regressors, factor models have identifying power, in that they can be used to create instrumental variables even when none of the observed variables are valid instruments.

The rest of the paper is organized as follows. In Section 2 we formally describe the triangular system with our factor structure, and discuss our main identification results for the parameters of 
interest in this model. Section 3 explores identification in more general factor structure models which involve multiple idiosyncratic errors, in a context where one has access to two continuous noisy measurements of the common unobserved factor. Finally, Section 4 concludes. The Supplementary Material collects the proofs of our results, derives and studies the asymptotic properties of a rank-based estimator for $\alpha_{0}$, explores its finite sample properties through some Monte Carlo simulation exercises, and shows the non-identification of the two-factor model with a compactsupported common factor and no continuous repeated measurements. Finally, we also establish the sharp identified set of $\alpha_{0}$ when the support condition for point-identification is violated.

Notation: throughout the paper we write $\mathbf{1}\{A\}$ to denote the usual indicator function that takes value 1 if event $A$ happens, and 0 otherwise. We also denote by $d(U)$ and $d(U \mid V)$ the lengths of the support of random variable $U$, and the conditional support of $U$ given $V$, respectively.

\section{$2 \quad$ Triangular Binary Model with Factor Structure}

\subsection{Set-up and Main Identification Result}

In this section we consider the identification of the following triangular binary model:

$$
\begin{aligned}
Y_{1} & =\mathbf{1}\left\{Z_{1}^{\prime} \lambda_{0}+Z_{3}^{\prime} \beta_{0}+\alpha_{0} Y_{2}-U>0\right\} \\
Y_{2} & =\mathbf{1}\left\{Z^{\prime} \delta_{0}-V>0\right\}
\end{aligned}
$$

where $Z \equiv\left(Z_{1}, Z_{2}\right)$ and $(U, V)$ is a pair of random shocks. $Z_{2}$ and $Z_{3}$ provide the exclusion restrictions in the model, and the distribution of $\left(Z_{2}, Z_{3}\right)$ is required to be nondegenerate conditional on $Z_{1}^{\prime} \lambda_{0}+Z_{3}^{\prime} \beta_{0}$. We further assume that the error terms $U$ and $V$ are jointly independent of $\left(Z_{1}, Z_{2}, Z_{3}\right)$. The endogeneity of $Y_{2}$ in (2.4) arises when $U$ and $V$ are not independent.

The above model, or minor variations of it, have often been considered in the recent literature. See for example, Vytlacil and Yildiz (2007), Abrevaya, Hausman, and Khan (2010), Klein, Shan, and Vella (2015), Vuong and Xu (2017), Khan and Nekipelov (2018) and references therein. A key parameter of interest in our paper and in the rest of the literature is $\alpha_{0}$. In this paper we provide conditions under which the parameters of interest are point-identified. As such, our analysis complements alternative partial-identification approaches that have been proposed in the context of triangular binary models. See, in particular, Chiburis (2010), Shaikh and Vytlacil (2011), and Mourifié (2015). ${ }^{3}$ As discussed in the aforementioned papers, the parameter $\alpha_{0}$ is difficult, if not impossible to identify and estimate without imposing parametric restrictions on the unobserved

\footnotetext{
${ }^{3}$ In Section $\mathrm{E}$ in the supplement, we establish the sharp identified set of $\alpha_{0}$ when the support condition for point-identification is violated. This result highlights that, except for the fact that the sign of $\alpha_{0}$ is identified, we generally cannot say much about the value of $\left|\alpha_{0}\right|$. Related work by Shaikh and Vytlacil (2011) also provides partial identification results for a triangular binary model. That the bounds for $\alpha_{0}$ are generally tighter in their analysis reflects the identifying power of the additional support restrictions that they impose.
} 
variables in the model, $(U, V)$.

The difficulty of identifying $\alpha_{0}$ in semi-parametric "distribution-free" models, and the sensitivity of its identification to misspecification in parametric models is what motivates the factor structure we add in this paper to the above model. Specifically, to allow for endogeneity in the form of possible non-zero correlation between $U$ and $V$, we augment the model with the following equation:

$$
U=\gamma_{0} V+\Pi
$$

where $\Pi$ is an unobserved random variable, assumed to be distributed independently of $\left(V, Z_{1}, Z_{2}, Z_{3}\right)$, and $\gamma_{0}$ is an additional unknown scalar parameter. Importantly, this type of factor structure always holds when the residuals of both equations are jointly normally distributed. Furthermore, this specification corresponds to the type of structure used in Independent Component Analysis (ICA), where $V$ and $\Pi$ are two mutually independent factors. This method has found many applications in various fields, including signal processing and image extraction; applications in economics include e.g., Hyvärinen and Oja (2000), Moneta, Hoyer, and Coad (2013) and Gourieroux, Monfort, and Renne (2017). While, in contrast to the ICA literature, the factors and the factor loadings are not the main objects of interest in our analysis, this dimension-reducing structure plays a key role in our identification results.

Our aim is to first explore identification of the parameters $\left(\alpha_{0}, \delta_{0}, \gamma_{0}, \beta_{0}, \lambda_{0}\right)$ under standard nonparametric regularity conditions on $(V, \Pi)$. Note that the parameter $\delta_{0}$ in the selection equation can be identified up to scale in various ways. See, for example, Klein and Spady (1993) and Han (1987), among others. We then impose the usual condition that one of $\delta_{0}$ 's coordinates is equal to one to fix the scale. For simplicity, for the rest of the paper, we denote $X \equiv Z^{\prime} \delta_{0}$ and assume $X$ is observed. We further define $X_{1} \equiv Z_{1}^{\prime} \lambda_{0}+Z_{3}^{\prime} \beta_{0}$. However, we cannot identify $\lambda_{0}$ and $\beta_{0}$ beforehand. We propose instead to identify them along with $\alpha_{0}$.

Our main identification result is based on the Assumptions A1-A4 we state below:

A1 The first coefficient of $\lambda_{0}$ is normalized to one so that $\lambda_{0}=\left(1, \lambda_{0,-1}^{T}\right)^{T}$. The parameter $\theta_{0} \equiv\left(\alpha_{0}, \gamma_{0}, \lambda_{0,-1}, \beta_{0}\right)$ is an element of a compact subset of $\Re^{d_{1}+d_{3}+1}$, where $d_{1}$ and $d_{3}$ are the dimensions of $Z_{1}$ and $Z_{3}$, respectively.

A2 The vector of unobserved variables, $(U, V, \Pi)$ is continuously distributed with support on a subset of $\Re^{3}$ and independently distributed of the vector $\left(Z_{1}, Z_{2}, Z_{3}\right)$. Furthermore, we assume that the unobserved random variables $\Pi, V$ are distributed independently of each other.

A3 $X$ is continuously distributed with absolute continuous density w.r.t. Lebesgue measure. Its density is bounded and bounded away from zero on any compact subset of its support.

A4 Let $Z_{1,-1}$ be all the coordinates of $Z_{1}$ except the first one, and $d=d_{1}+d_{3}+1$. There exist $2 d$ 
vectors $\left\{z_{1}^{(l)}, z_{3}^{(l)}, x^{(l)}\right\}_{l=1}^{d}$ and $\left\{\tilde{z}_{1}^{(l)}, \tilde{z}_{3}^{(l)}, \tilde{x}^{(l)}\right\}_{l=1}^{d}$ in the joint support of $\left(Z_{1}, Z_{3}, X\right)$ such that

$$
\alpha_{0}+\left(z_{1,-1}^{(l)}-\tilde{z}_{1,-1}^{(l)}\right)^{\prime} \lambda_{0,-1}+\left(z_{3}^{(l)}-\tilde{z}_{3}^{(l)}\right)^{\prime} \beta_{0}-\gamma_{0}\left(x^{(l)}-\tilde{x}^{(l)}\right)=\tilde{z}_{1,1}^{(l)}-z_{1,1}^{(l)}, l=1, \cdots, d
$$

and $\operatorname{rank}(\mathcal{M})=d$, where

$$
\mathcal{M}=\left(\begin{array}{ccc}
1 & \cdots & 1 \\
z_{1,-1}^{(1)}-\tilde{z}_{1,-1}^{(1)} & \cdots & z_{1,-1}^{(d)}-\tilde{z}_{1,-1}^{(d)} \\
z_{3}^{(1)}-\tilde{z}_{3}^{(1)} & \cdots & z_{3}^{(d)}-\tilde{z}_{3}^{(d)} \\
x^{(1)}-\tilde{x}^{(1)} & \cdots & x^{(d)}-\tilde{x}^{(d)}
\end{array}\right) .
$$

Before turning to our main identification result, a couple of remarks are in order.

Remark 2.1. The first part of Assumption A1 is a standard scale normalization. Assumption A2 is also standard in this literature. The assumption that the instruments are independent of the unobservables can also be found in, among others, Abrevaya, Hausman, and Khan (2010), Vytlacil and Yildiz (2007), Klein, Shan, and Vella (2015), and Khan and Nekipelov (2018). The assumption of independence between $\Pi$ and $V$ is also made in Bai and $N g$ (2002) and Carneiro, Hansen, and Heckman (2003).

Remark 2.2. Assumptions $A 3$ and A4 impose some restrictions on the distributions of the covariates entering the selection and outcome equations, respectively. Specifically, Assumption A3 requires one component of the covariates $Z$ entering the selection equation to be continuously distributed, which is often required in models with discrete outcomes. In contrast, Assumption A4 only requires some variation of $\left(Z_{1}, Z_{3}\right)$. In particular, the distribution of $\left(Z_{1}, Z_{3}\right)$ cannot be degenerate but is allowed to be discrete. This assumption can be interpreted as a full rank condition, which ensures that the system of linear equations that delivers point identification has a unique solution.

We now turn to our main identification result, Theorem 2.1, which concludes that under our stated conditions and our factor structure we can attain point identification of the vector of parameters $\theta_{0}$.

Theorem 2.1. Under Assumptions A1-A4, $\theta_{0}$ is point identified.

An important takeaway from this result, which we discuss further in Subsection 2.2 below, is that imposing the factor structure (2.6) yields point-identification under weaker support conditions when compared to the existing literature, and does not require a second exclusion restriction either. In particular, our model delivers point-identification of the parameters of interest even in situations where all of the regressors from the outcome equation are discrete. This indicates that, from the selection equation combined with the factor structure that we impose here, we can overturn the non-identification result of Bierens and Hartog (1988) which would apply to the outcome equation alone. 
The proof of Theorem 2.1, which is reported in Section H in the Supplementary Appendix, relies on the fact that, for two observations $\left(Z_{1}, Z_{3}, X\right)$ and $\left(\tilde{Z}_{1}, \tilde{Z}_{3}, \tilde{X}\right)$,

$$
\begin{aligned}
& \partial_{x} P^{11}\left(Z_{1}, Z_{3}, X\right) / f_{V}(X)+\partial_{x} P^{10}\left(\tilde{Z}_{1}, \tilde{Z}_{3}, \tilde{X}\right) / f_{V}(\tilde{X})=0 \\
\Longleftrightarrow & \alpha_{0}+\left(Z_{1}-\tilde{Z}_{1}\right)^{\prime} \lambda_{0}+\left(Z_{3}-\tilde{Z}_{3}\right)^{\prime} \beta_{0}-\gamma_{0}(X-\tilde{X})=0,
\end{aligned}
$$

where $f_{V}(\cdot)$ is the pdf. of $V$, which is identified over the support of $X$, and $P^{i j}\left(z_{1}, z_{3}, x\right) \equiv$ $\operatorname{Prob}\left(Y_{1}=i, Y_{2}=j \mid Z_{1}=z_{1}, Z_{3}=z_{3}, X=x\right)\left(\partial_{x} P^{i j}\left(z_{1}, z_{3}, x\right)\right)$ denote the choice probability (partial derivative of the $i j$-choice probability with respect to the third argument), which are both identified from the data.

Remark 1. This identification result can be extended to the case of a separable nonparametric factor model. Namely, consider the following relationship between unobserved components:

$$
U=g_{0}(V)+\tilde{\Pi}
$$

where $\tilde{\Pi}$ is an unobserved random variable assumed to be distributed independently of $V$ and all instruments. $g_{0}(\cdot)$ is an unknown function assumed to satisfy standard smoothness conditions. The parameter of interest is $\left(\alpha_{0}, \lambda_{0}, \beta_{0}\right)$, but now the unknown nuisance parameter in the factor equation is infinite dimensional. By replacing $\gamma_{0} X$ by $g_{0}(X)$ in (2.7), we have

$$
\begin{aligned}
& \partial_{x} P^{11}\left(Z_{1}, Z_{3}, X\right) / f_{V}(X)+\partial_{x} P^{10}\left(\tilde{Z}_{1}, \tilde{Z}_{3}, \tilde{X}\right) / f_{V}(\tilde{X})=0 \\
\Longleftrightarrow & \alpha_{0}+\left(Z_{1}-\tilde{Z}_{1}\right)^{\prime} \lambda_{0}+\left(Z_{3}-\tilde{Z}_{3}\right)^{\prime} \beta_{0}-\left(g_{0}(X)-g_{0}(\tilde{X})\right)=0 .
\end{aligned}
$$

One can then establish identification after modifying the rank condition A4 by replacing $\gamma_{0}\left(x^{(l)}-\tilde{x}^{(l)}\right)$ by $g_{0}\left(x^{(l)}\right)-g_{0}\left(\tilde{x}^{(l)}\right)$.

\subsection{Connection with Prior Literature}

We now discuss in detail how our setup and main identification result relates to the existing literature.

In a related work, Han and Vytlacil (2017) consider the identification of a generalized bivariate Probit model. ${ }^{4}$ Our linear factor structure and the one-parameter copula model considered in Han and Vytlacil (2017) are not nested by each other. First, note that based on the factor structure, we can recover $F_{\Pi}$, the distribution of $\Pi$, as a function of $\left(F_{U}, F_{V}, \gamma_{0}\right)$ by deconvolution. We can then write the copula of $(U, V)$ as

$$
F_{U, V}\left(F_{U}^{-1}(u), F_{V}^{-1}(v)\right)=\int_{-\infty}^{F_{V}^{-1}(v)} F_{\Pi}\left(F_{U}^{-1}(u)-\gamma_{0} w ; F_{U}, F_{V}, \gamma_{0}\right) f_{V}(w) d w=C\left(u, v ; F_{U}, F_{V}, \gamma_{0}\right) .
$$

\footnotetext{
${ }^{4}$ See also recent work by Han and Lee (2019) who study semiparametric estimation and inference in the framework considered by Han and Vytlacil (2017).
} 
The copula depends not only on $\gamma_{0}$ but also on two infinite dimensional parameters $\left(F_{U}, F_{V}\right)$. Thus, unlike Han and Vytlacil (2017), our factor structure cannot be characterized by a one-parameter copula. In addition, in order to achieve identification, Han and Vytlacil (2017) first nonparametrically identify the two marginals by assuming the existence of a full support regressor that is common to both equations. ${ }^{5}$ In contrast, our approach does not rely on the existence of such a regressor. Under the factor structure assumed in our analysis, we bypass the nonparametric identification of the marginals as a whole and directly consider the identification of the structural parameters. It follows that our model cannot be nested by the one-parameter copula model considered by Han and Vytlacil (2017). On the other hand, there exist one-parameter copula models that cannot be decomposed into linear factor structures. ${ }^{6}$ This implies that our model does not nest Han and Vytlacil (2017) either.

Our analysis also relates to Vytlacil and Yildiz (2007) and Vuong and Xu (2017), who consider the identification of $\alpha_{0}$ in a triangular binary model. Our identification result, however, differs from theirs in important ways. Namely, denote $X=Z^{\prime} \delta_{0}=Z_{1}^{\prime} \delta_{1,0}+Z_{2}^{\prime} \delta_{2,0}$. Then, Assumption A4 implies that we can find a pair of observations $\left(z_{1}, z_{2}, z_{3}\right)$ and $\left(\tilde{z}_{1}, \tilde{z}_{2}, \tilde{z}_{3}\right)$ such that

$$
z_{1}^{\prime} \lambda_{0}+z_{3}^{\prime} \beta_{0}+\alpha_{0}-\gamma_{0}\left(z_{1}^{\prime} \delta_{1,0}+z_{2}^{\prime} \delta_{2,0}\right)=\tilde{z}_{1}^{\prime} \lambda_{0}+\tilde{z}_{3}^{\prime} \beta_{0}-\gamma_{0}\left(\tilde{z}_{1}^{\prime} \delta_{1,0}+\tilde{z}_{2}^{\prime} \delta_{2,0}\right)
$$

In contrast, using our notation, Vytlacil and Yildiz (2007) require that one can find a pair of observations $\left(z_{1}, z_{2}, z_{3}\right)$ and $\left(\tilde{z}_{1}, \tilde{z}_{2}, \tilde{z}_{3}\right)$ such that $z^{\prime} \delta_{0}=\tilde{z}^{\prime} \delta_{0}$ and

$$
z_{1}^{\prime} \lambda_{0}+z_{3}^{\prime} \beta_{0}+\alpha_{0}=\tilde{z}_{1}^{\prime} \lambda_{0}+\tilde{z}_{3}^{\prime} \beta_{0}
$$

Vuong and $\mathrm{Xu}$ (2017) do not assume the existence of $Z_{3}$. In our binary outcome setup, the functions $h(0, x, \tau)$ and $h(1, x, \tau)$ defined in Vuong and $\mathrm{Xu}(2017)$ are equal to $1\left\{x+F_{-U}^{-1}(\tau) \geq 0\right\}$ and $1\left\{x+\alpha+F_{-U}^{-1}(\tau) \geq 0\right\}$, respectively, where $x=z_{1}^{\prime} \lambda_{0}$ and $F_{-U}$ is the CDF of $-U$. Then, Vuong and $\mathrm{Xu}$ (2017, Assumption C'(ii)) requires that we can find $z_{1}$ and $\tilde{z}_{1}$ in the support of $Z_{1}$ so that for any $\tau_{1}, \tau_{2}$, if $1\left\{\tilde{z}_{1}^{\prime} \lambda_{0}+F_{-U}^{-1}\left(\tau_{1}\right) \geq 0\right\}=1\left\{\tilde{z}_{1} \lambda_{0}+F_{-U}^{-1}\left(\tau_{2}\right) \geq 0\right\}$, then $1\left\{z_{1}^{\prime} \lambda_{0}+\alpha_{0}+F_{-U}^{-1}\left(\tau_{1}\right) \geq\right.$ $0\}=1\left\{z_{1} \lambda_{0}+\alpha_{0}+F_{-U}^{-1}\left(\tau_{2}\right) \geq 0\right\}$. Provided that the support of $U$ nests the supports of $Z_{1}^{\prime} \lambda_{0}$ and

\footnotetext{
${ }^{5}$ Han and Vytlacil (2017) establish their identification of the coefficient on the endogeneous regressor (Theorems 4.2 and 5.1) under the assumption that the marginal distributions $F_{\varepsilon}$ and $F_{\nu}$ are known. Then, they verify this condition by showing the identification of these two marginal distributions using large support common regressors.

${ }^{6}$ For instance, suppose that $(U, V)$ has a Gaussian copula with correlation $\rho$, and that the marginal distributions of $U$ and $V$ are uniform [0,1]. It then follows that, denoting by $\Phi($.$) the standard normal cdf., \left(\Phi^{-1}(U), \Phi^{-1}(V)\right)$ is bivariate normal with correlation $\rho$, which in turn yields the following non-linear relationship between $U$ and $V$ : $U=\Phi\left(\rho \Phi^{-1}(V)+W\right)$, where $W$ is normally distributed and independent from $V$.
} 
$Z_{1}^{\prime} \lambda_{0}+\alpha_{0}$, Vuong and $\mathrm{Xu}\left(2017\right.$, Assumption $\left.\mathrm{C}^{\prime}(\mathrm{ii})\right)$ is then equivalent to: ${ }^{7}$

$$
z_{1}^{\prime} \lambda_{0}+\alpha_{0}=\tilde{z}_{1}^{\prime} \lambda_{0}
$$

Several remarks are in order. First, note that sufficient support conditions for the restrictions $(2.10)-(2.12)$ are $d\left(Z_{1}^{\prime} \lambda_{0}+Z_{3}^{\prime} \beta_{0}-Z^{\prime} \delta_{0} \gamma_{0}\right) \geq\left|\alpha_{0}\right|, d\left(Z_{1}^{\prime} \lambda_{0}+Z_{3}^{\prime} \beta_{0} \mid Z^{\prime} \delta_{0}\right) \geq\left|\alpha_{0}\right|$, and $d\left(Z_{1}^{\prime} \lambda_{0} \mid Z^{\prime} \delta_{0}\right) \geq$ $\left|\alpha_{0}\right|$ with a positive probability, respectively. These three support conditions are such that

$$
d\left(Z_{1}^{\prime} \lambda_{0}+Z_{3}^{\prime} \beta_{0}-Z^{\prime} \delta_{0} \gamma_{0}\right) \geq d\left(Z_{1}^{\prime} \lambda_{0}+Z_{3}^{\prime} \beta_{0} \mid Z^{\prime} \delta_{0}\right) \geq d\left(Z_{1}^{\prime} \lambda_{0} \mid Z^{\prime} \delta_{0}\right)
$$

where the first and second inequalities are strict if $Z_{2}$ and $Z_{3}$ have at least one continuous component, respectively. Importantly, we show in Section E of the Supplement that for a version of the triangular binary model with univariate $Z_{2}$ and $Z_{3}$ and no common regressor $Z_{1}$, the support condition $d\left(Z_{1}^{\prime} \lambda_{0}+Z_{3}^{\prime} \beta_{0} \mid Z^{\prime} \delta_{0}\right) \geq\left|\alpha_{0}\right|$ is actually also necessary to the identification of the model without factor structure. This implies that by imposing our factor structure, one can identify values of $\alpha_{0}$ in a region that cannot be identified in the model considered by Vytlacil and Yildiz (2007). Such region is characterized in Section E of the Supplement.

Second, it directly follows from these support conditions that, in the presence of a factor model and in contrast to both Vytlacil and Yildiz (2007) and Vuong and Xu (2017), variation in $Z_{2}$ helps in the identification of $\alpha_{0}$. In that sense, the factor model allows to restore the intuition from standard IV approaches in linear models that variation in the instrument $Z_{2}$ is critical to the identification of the parameters of the outcome equation. Related to this, the support of $Z_{2}$ plays an important role in our identification analysis. In particular, if $Z_{2}$ is discrete, our identification strategy requires sufficient variation in the variables in the outcome equation, namely $Z_{1}$ and $Z_{3}$. In this case, our support requirement is equivalent to that assumed by Vytlacil and Yildiz (2007).

Third, another important aspect of Assumption A4 is that it does not impose any constraint on the variables from the outcome equation. Specifically, consider a case where the outcome equation does not contain a variable that is excluded from the selection equation (i.e., $\beta_{0}=0$ ), the regressor that is common to both equations, $Z_{1}$, is scalar and binary, and where $\lambda_{0}=1$. In this case, one can show that the identifying support conditions associated with Vytlacil and Yildiz (2007) (2.11) and Vuong and $\mathrm{Xu}$ (2017) (2.12) generally fail to hold, except for a finite set of values $\alpha_{0} \in\{-1,0,1\}$. In contrast, our support restriction (2.10) holds under more general conditions: without any restriction on $\alpha_{0}$ if one element of $Z_{2}$ is continuous with large support, and on a continuum of possible values for $\alpha_{0}$ if one element of $Z_{2}$ is continuous with bounded support. In that sense, the factor structure replaces the need for a continuous component in $\left(Z_{1}, Z_{3}\right)$ in the outcome equation.

\footnotetext{
${ }^{7}$ To see this, note that if, say, $z_{1}^{\prime} \lambda_{0}+\alpha_{0}>\tilde{z}_{1}^{\prime} \lambda_{0}$, then we can find $\tau_{1}, \tau_{2}$ such that $-z_{1}^{\prime} \lambda_{0}-\alpha_{0} \leq F_{-U}^{-1}\left(\tau_{1}\right)<-\tilde{z}_{1}^{\prime} \lambda_{0}$ and $F_{-U}^{-1}\left(\tau_{2}\right)<-z_{1}^{\prime} \lambda-\alpha_{0}<-\tilde{z}_{1}^{\prime} \lambda_{0}$. This violates the above requirement, and thus, shows that Vuong and Xu (2017, Assumption C'(ii)) implies (2.12). On the other hand, if $z_{1}^{\prime} \lambda_{0}+\alpha_{0}=\tilde{z}_{1}^{\prime} \lambda_{0}$, then Vuong and Xu (2017, Assumption C'(ii)) holds trivially.
} 
Finally, at a high level, our identification strategy shares similarities with the Local Instrumental Variable (LIV) approach that has been proposed by Heckman and Vytlacil (2005) and further discussed by Carneiro and Lee (2009). In particular, our identifying restriction (2.7) can be alternatively derived from a local IV strategy applied to a potential outcomes model characterized by $Y_{1}\left(y_{2}\right)=\mathbf{1}\left\{Z_{1}^{\prime} \lambda_{0}+Z_{3}^{\prime} \beta_{0}+\alpha_{0} y_{2}-U>0\right\}$, with treatment given by $Y_{2}=\mathbf{1}\left\{Z^{\prime} \delta_{0}-V>0\right\}$. In contrast to the LIV literature though, we focus in our analysis on the structural parameter $\alpha_{0}$ rather than on the marginal treatment effects. Our identification result shows that, by leveraging the identifying power of the factor structure, one can identify $\alpha_{0}$ under weaker support restrictions than in the prior literature. In particular, our strategy makes it possible to use variation in $X=Z^{\prime} \delta_{0}$ to identify $\alpha_{0}$, even when all the components of $Z_{1}$ and $Z_{3}$ are discrete. $^{8}$

\section{Extended Factor Structure in the presence of Continuous Mea- surements}

Up until now we have proposed identification and estimation results for a triangular system with a particular factor structure. A disadvantage of this structure is that it only includes one idiosyncratic shock $(\Pi)$. We consider below an extension that addresses this limitation.

Namely, we consider the following model:

$$
\begin{aligned}
& Y_{1}=\mathbf{1}\left\{X_{1}+\alpha_{0} Y_{2}-U \geq 0\right\} \\
& Y_{2}=\mathbf{1}\{X-V \geq 0\}
\end{aligned}
$$

where $X_{1}=Z_{1}^{\prime} \lambda_{0}+Z_{3}^{\prime} \beta_{0}, X=Z^{\prime} \delta_{0}, U=\gamma_{0} W+\eta_{1}, V=W+\eta_{2}$, and $\left(W, \eta_{1}, \eta_{2}\right)$ are mutually independent. Recall that, following the arguments in Section 2.1 above, we assume that $X$ is observed. In addition, we assume two auxiliary continuous measurements

$$
\begin{aligned}
& Y_{3}=\nu_{0} W+\eta_{3} \\
& Y_{4}=\sigma_{0} W+\eta_{4},
\end{aligned}
$$

where $\left(W, \eta_{1}, \eta_{2}, \eta_{3}, \eta_{4}\right)$ are mutually independent, and $\nu_{0} \neq 0$.

Our identification result is based on the following assumptions:

B0 The first coefficient of $\lambda_{0}$ is normalized to one so that $\lambda_{0}=\left(1, \lambda_{0,-1}^{T}\right)^{T}$. The parameter $\theta_{0} \equiv\left(\alpha_{0}, \gamma_{0}, \lambda_{0,-1}, \beta_{0}, \nu_{0}, \sigma_{0}\right)$ is an element of a compact subset of $\Re^{d_{1}+d_{3}+3}$, where $d_{1}$ and $d_{3}$ are the dimensions of $Z_{1}$ and $Z_{3}$, respectively. The vector of unobservables in the outcome

\footnotetext{
${ }^{8}$ An alternative approach to identifying this parameter can be found in Lewbel (2000). In his approach a second equation to model the endogenous variable is not needed, nor is the factor structure we impose. However, he imposes a strong support condition on a variable like $Z_{3}$ requiring that it exceeds the length of the unobservable $U$.
} 
and selection equations $\left(W, \eta_{1}, \eta_{2}, \eta_{3}\right)$ are independently distributed of the vector $\left(Z_{1}, Z_{2}, Z_{3}\right)$. Both $\eta_{1}$ and $\eta_{2}$ are continuously distributed.

B1 $\gamma_{0} \neq 0 . \quad X$ is continuously distributed with absolute continuous density w.r.t. Lebesgue measure over the whole real line, conditionally on $Z_{1}$ and $Z_{3}$. The unconditional density of $X$ is bounded and bounded away from zero on any compact subset of its support.

B2 $W$ is not normally distributed or both $\eta_{3}$ and $\eta_{4}$ do not have a Gaussian component.

B3 $E\left(\eta_{3}\right)=E\left(\eta_{4}\right)=0, E\left(\left|\eta_{3}\right|\right)<\infty$, and $E\left(\left|\eta_{4}\right|\right)<\infty$.

B4 $E\left(\exp \left(i \zeta \eta_{2}\right)\right), E\left(\exp \left(i \zeta \eta_{3}\right)\right)$, and $E\left(\exp \left(i \zeta \eta_{4}\right)\right)$ do not vanish for any $\zeta \in \Re$, where $i=\sqrt{-1}$.

B5 $E(\exp (i \zeta W)) \neq 0$ for all $\zeta$ in a dense subset of $\Re$.

B6 The distributions of $W, \eta_{2}$, and $\eta_{3}$ admit uniformly bounded densities $f_{W}(\cdot), f_{\eta_{2}}(\cdot)$, and $f_{\eta_{3}}(\cdot)$ with respect to the Lebesgue measure that are supported on an interval (which may be infinite), respectively.

B7 Let $Z_{1,-1}$ be all the coordinates of $Z_{1}$ except the first one, and $d=d_{1}+d_{3}+1$. There exist $2 d$ vectors $\left\{z_{1}^{(l)}, z_{3}^{(l)}\right\}_{l=1}^{d}$ and $\left\{\tilde{z}_{1}^{(l)}, \tilde{z}_{3}^{(l)}\right\}_{l=1}^{d}$ in the joint support of $\left(Z_{1}, Z_{3}\right)$ and $\left\{w^{(l)}\right\}_{l=1}^{d},\left\{\tilde{w}^{(l)}\right\}_{l=1}^{d}$ such that

$$
\alpha_{0}+\left(z_{1,-1}^{(l)}-\tilde{z}_{1,-1}^{(l)}\right)^{\prime} \lambda_{0,-1}+\left(z_{3}^{(l)}-\tilde{z}_{3}^{(l)}\right)^{\prime} \beta_{0}-\gamma_{0}\left(w^{(l)}-\tilde{w}^{(l)}\right)=\tilde{z}_{1,1}^{(l)}-z_{1,1}^{(l)}, l=1, \cdots, d
$$

and $\operatorname{rank}(\mathcal{M})=d$, where

$$
\mathcal{M}=\left(\begin{array}{ccc}
1 & \cdots & 1 \\
z_{1,-1}^{(1)}-\tilde{z}_{1,-1}^{(1)} & \cdots & z_{1,-1}^{(d)}-\tilde{z}_{1,-1}^{(d)} \\
z_{3}^{(1)}-\tilde{z}_{3}^{(1)} & \cdots & z_{3}^{(d)}-\tilde{z}_{3}^{(d)} \\
w^{(1)}-\tilde{w}^{(1)} & \cdots & w^{(d)}-\tilde{w}^{(d)}
\end{array}\right)
$$

We now discuss these assumptions, before turning to the identification result. First, Assumption B0 is similar to Assumptions A1 and A2. We only need one of the idiosyncratic errors in the continuous measurements to be independent of the covariates because the other one is used to identify the distribution of the common factor $W$ only. Second, as we assume in Assumption B1 that $\gamma_{0} \neq 0$ and $X$ has full support, the support condition

$$
d\left(Z_{1}^{\prime} \lambda_{0}+Z_{3}^{\prime} \beta_{0}-\gamma_{0} X\right) \geq\left|\alpha_{0}\right|
$$

holds automatically. The full support condition of $X$ is necessary to identify the density of $V$, which is further used to identify the distribution of $\eta_{2}$. Assumption B1 reinforces this condition by supposing that $X$ has full support conditional on $Z_{1}$ and $Z_{3}$, which is needed to identify the parameters from the outcome equation in a second step. Since $X=Z^{\prime} \delta_{0}$ with $Z=\left(Z_{1}, Z_{2}\right)$, this is 
in turn equivalent to $Z_{2}$ having full support conditional on $Z_{1}$ and $Z_{3}$. Third, Assumptions B2-B6 imply Assumptions 1 to 4 in $\mathrm{Hu}$ and Schennach (2013). In practice we add the condition that the characteristic function of $\eta_{2}$ does not vanish, which is used for the deconvolution arguments in the proof of Theorem 3.1. We refer the reader to Hu and Schennach (2013) for more discussions of these assumptions. ${ }^{9}$

Theorem 3.1. If (K.31)-(3.3) and Assumptions B0-B7 hold, then $\theta_{0}$ are identified.

The proof of Theorem 3.1 can be found in Section I of the Supplement. Several remarks are in order. First, while we allow for a more general factor structure on the unobservables $U$ and $V$, we also depart from our baseline specification by supposing that we have access to two continuous noisy measurements of the common factor $W$. This is a standard requirement in the nonparametric measurement error literature (Hu and Schennach, 2008). Besides, assuming access to a set of (selection-free) noisy measurements of the unobserved factors is also very standard in the evaluation literature. See, among many others, Carneiro, Hansen, and Heckman (2003), Heckman and Navarro (2007), Heckman and Vytlacil (2007a), and Cunha, Heckman, and Schennach (2010). For instance, in applications in labor economics, the unobserved factor $W$ often captures individual ability. This would apply, for example, to the evaluation of the effect of college employment $\left(Y_{2}\right)$ on college graduation $\left(Y_{1}\right)$. In these cases, cognitive skill measurements, such as the ASVAB test components that are available in the NLSY79 and NLSY97 surveys, are natural and often used candidates for these types of continuous measurements (Ashworth, Hotz, Maurel, and Ransom, 2020).

Second, as is clear from the proof of Theorem 3.1, the key purpose of the continuous measurements is to identify the distribution of the common factor $W$. While we assume in this section that the measurement equations are linear, it is possible to identify $\theta_{0}$ with a more general nonlinear system of continuous measurements, provided that the researcher has access to at least three such measurements. One can then combine Theorem 2 in Cunha, Heckman, and Schennach (2010) (Section 3.3, pp. 894-895), that yields identification of the distribution of $W$, with the proof of Theorem 3.1 in order to show identification of $\theta_{0}$ for the case of nonlinear auxiliary measurements. Assuming access to a set of at least three measurements also makes it possible to relax the nonnormality requirement imposed in Assumption B2.

Third, similar to the earlier discussions in Remark 2.2 and Section 2.2, Assumption B7 may still hold even when $Z_{3}$ is an empty set and $Z_{1}$ is discrete, since $W$ is assumed to have full support. In such a case, identification primarily relies on the factor structure and the variation of the covariates in the selection equation, rather than that in the outcome equation. In this respect, this identification result is similar in spirit to Theorem 2.1 and different from the existing identification results in the literature for triangular binary models, e.g., Vytlacil and Yildiz (2007) and Vuong and Xu (2017). More generally, in Section E.2 in the supplement we establish that the

\footnotetext{
${ }^{9}$ Note that $\mathrm{Hu}$ and Schennach (2013, Assumptions 5 and 6$)$ hold automatically in our model with $\nu_{0} \neq 0$.
} 
factor model provides identification restrictions that are not otherwise available. ${ }^{10}$

\section{Conclusion}

In this paper, we explore the identifying power of linear factor structures in the context of simultaneous binary response models. We impose two alternative types of factor structures on the unobservables of the model. The first setup is a natural distribution-free extension of the bivariate Probit model, while the second model corresponds to a standard linear factor model with one common factor and two equation-specific idiosyncratic shocks. We establish that both factor models have identifying power in that they make it possible to relax some of the exclusion and support conditions typically required for identification in this class of models (Vytlacil and Yildiz, 2007). Overall, our analysis complements results obtained by Bai and Ng (2010) in the context of a linear regression model with endogenous regressors, and, more generally, adds to our understanding of the identifying power of factor models, beyond their well known usefulness to recover the joint distribution of potential outcomes from the marginal distributions.

The work here opens areas for future research. The factor structure we assume could prove useful in more general nonlinear models. For instance, non-triangular discrete systems have shown to be an effective way to model entry games in the empirical industrial organization literature- see, for example, Tamer (2003). However, as shown in Khan and Nekipelov (2018), identification of structural parameters in these models can be even more challenging than for the triangular model considered in this paper. It would be useful to determine if factor structures on the unobservables could alleviate this problem. We leave this open question to future work.

\section{References}

Abbring, J., And J. Heckman (2007): "Econometrics Evaluation of Social Programs, Part III:

Distributional Treatment Effects, Dynamic Treatment Effects, Dynamic Discrete Choice, and General Equilibrium Policy Evaluation," in Handbook of Econometrics, Vol. 6B, ed. by J. J. Heckman, and E. E. Leamer. North Holland.

Abowd, J. M., and D. CARD (1989): "On the Covariance Structure of Earnings and Hours Changes," Econometrica, 57(2), 411-445.

\footnotetext{
${ }^{10}$ Specifically, we consider a version of the model (K.31), where we do not impose the factor structure and allow for an arbitrary (unknown to econometricians) dependence structure across the unobservables of the model. In this case, we show non-identification of $\alpha_{0}$ as long as $\left|\alpha_{0}\right|>b-a$, where $[a, b]$ denotes the conditional support of $X_{1}$ given $X$ and, consistent with our Assumption B1, $X$ has full support on the real line. However, by imposing the factor structure (and other conditions implied by B0-B7), Theorem 3.1 shows that $\alpha_{0}$ is identified for this model even when $\left|\alpha_{0}\right|>b-a$.
} 
Abrevaya, J., J. Hausman, and S. Khan (2010): "Testing for Causal Effects in a Generalized Regression Model with Endogenous Regressors," Econometrica, 78(6), 2043-2061.

Ashworth, J., V. J. Hotz, A. Maurel, and T. Ransom (2020): "Changes Across Cohorts in Wage Returns to Schooling and Early Work Experiences," forthcoming, Journal of Labor Economics.

BAI, J., AND S. NG (2002): "Determining the Number of Factors in Approximate Factor Models," Econometrica, 70(1), 191-221.

BAI, J., And S. NG (2010): "Instrumental Variable Estimation in a Data Rich Environment," Econometric Theory, 26(6), 1577-1606.

Bierens, H., and J. Hartog (1988): "Non-Linear Eegression with Discrete Explanatory Variables, with an Application to the Earnings Function," Journal of Econometrics, 38(3), 269-299.

Bonhomme, S., And J.-M. Robin (2010): "Generalized Non-Parametric Deconvolution with an Application to Earnings Dynamics," Review of Economic Studies, 77(2), 491-533.

Butucea, C., And C. Matias (2005): "Minimax estimation of the noise level and of the deconvolution density in a semiparametric convolution model," Bernoulli, 11(2), 309-340.

Carneiro, P., K. Hansen, and J. J. Heckman (2003): "Estimating Distributions of Treatment Effects with an Application to the Returns to Schooling and Measurement of the Effects of Uncertainty on College Choice," International Economic Review, 44(2), 361-422.

Carneiro, P., and S. Lee (2009): "Estimating distributions of potential outcomes using local instrumental variables with an application to changes in college enrollment and wage inequality," Journal of Econometrics, 149(2), 191-208.

Chen, S., S. Khan, and X. TAng (2016): "On the Informational Content of Special Regressors in Heteroskedastic Binary Response Models," Journal of Econometrics, 193, 162-182.

Chesher, A. (2005): "Nonparametric identification under discrete variation," Econometrica, $73(5), 1525-1550$.

Chiburis, R. (2010): "Semiparametric Bounds on Treatment Effects," Journal of Econometrics, $159(2), 267-275$.

Cunha, F., J. J. Heckman, and S. M. Schennach (2010): "Estimating the Technology of Cognitive and Noncognitive Skill Formation," Econometrica, 78(3), 883-931.

Geary, R. (1942): "Inherent relations between random variables," Proceedings of the Royal Irish Academy, 47, 63-76. 
Gourieroux, C., A. Monfort, and J.-P. Renne (2017): "Statistical inference for independent component analysis: Application to structural VAR models," Journal of Econometrics, 196(1), $111-126$.

HAN, A. (1987): "Non-parametric analysis of a generalized regression model: The maximum rank correlation estimator," Journal of Econometrics, 35(2-3), 303-316.

Han, S., And S. LeE (2019): "Estimation in a Generalization of a Bivariate Probit Models with Dummy Endogenous Regressors," Journal of Applied Econometrics, 34(6), 994-1015.

HAN, S., AND E. J. Vytlacil (2017): "Identification in a generalization of bivariate probit models with endogenous regressors," Journal of Econometrics, 199(1), 63-73.

Heckman, J., and S. Navarro (2007): "Dynamic Discrete Choice and Dynamic Treatment Effects," Journal of Econometrics, 136(2), 341-396.

Heckman, J. J., J. Humphries, and G. Veramendi (2018): "Returns to Education: The Causal Effects of Education on Earnings, Health, and Smoking," Journal of Political Economy, 126, S197-S246.

Heckman, J. J., And E. Vytlacil (2005): "Structural equations, treatment effects, and econometric policy evaluation 1," Econometrica, 73(3), 669-738.

Heckman, J. J., And E. J. Vytlacil (2007a): "Econometric evaluation of social programs, part I: Causal models, structural models and econometric policy evaluation," Handbook of econometrics, $6,4779-4874$.

(2007b): "Econometric evaluation of social programs, part II: Using the marginal treatment effect to organize alternative econometric estimators to evaluate social programs, and to forecast their effects in new environments," Handbook of econometrics, 6, 4875-5143.

Henderson, D., Q. Li, C. Parmeter, and S. Yao (2015): "Gradient-based Smoothing Parameter Selection for Nonparametric Regression Estimation," Journal of Econometrics, 184, 233-241.

Horowitz, J. (1992): "A Smoothed Maximum Score Estimator for the Binary Response Model," Econometrica, 60(3).

Hu, Y., And S. M. Schennach (2008): "Instrumental variable treatment of nonclassical measurement error models," Econometrica, 76(1), 195-216.

Hu, Y., And S. M. Schennach (2013): "Nonparametric identification and semiparametric estimation of classical measurement error models without side information," Journal of the American Statistical Association, 108(501), 177-186.

Hyvärinen, A., AND E. OJA (2000): Independent component analysis: algorithms and applications, vol. 13. Elsevier. 
Jochmans, K. (2013): "Pairwise-comparison estimation with nonparametric controls," Econometrics Journal, 16, 340-372.

Khan, S. (2001): "Two Stage Rank Estimation of Quantile Index Models," Journal of Econometrics, 100, 319-355.

Khan, S., And D. NeKIPElov (2018): "Information structure and statistical information in discrete response models," Quantitative Economics, 9(2), 995-1017.

Khan, S., And E. TAmer (2018): "Discussion of "Simple Estimators for Invertible Index Models" by Ahn et al.," Journal of Business 83 Economic Statistics, 36, 11-15.

Klein, R., C. Shan, and F. Vella (2015): "Estimation of marginal effects in semiparametric selection models with binary outcomes," Journal of Econometrics, 185(1), 82-94.

Klein, R., and R. Spady (1993): "An Efficient Semiparametric Estimator for Binary Response Model," Econometrica, 61(2), 387-421.

Lewbel, A. (2000): "Semiparametric Qualitative Response Model Estimation with Unknown Heteroscedasticity or Instrumental Variables," Journal of Econometrics, 97(1), 145-177.

Lewbel, A., S. M. Schennach, and L. Zhang (2020): "Identification of a Triangular Two Equation System Without Instruments," Working Paper.

Moneta, A., D. E. P. O. Hoyer, And A. Coad (2013): "Causal inference by independent component analysis: Theory and applications," Oxford Bulletin of Economics and Statistics, 75(5), 705-730.

MourifiÉ, I. (2015): "Sharp Bounds on Treatment Effects in a Binary Triangular System," Journal of Econometrics, 187(1), 74-81.

Newey, W., and D. McFadden (1994): "Large Sample Estimation and Hypothesis Testing," in Handbook of Econometrics, Vol. 4, ed. by R. Engle, and D. McFadden. North Holland.

Powell, J., J. Stock, and T. Stoker (1989): "Semiparametric Estimation of Index Coefficients," Econometrica, pp. 1403-1430.

Reiersol, O. (1950): "Identifiability of a Linear Relation Between Variables Which are Subject to Error," Econometrica, 18(4), 375-389.

Shaikh, A. M., and E. Vytlacil (2011): "Partial Identification in Triangular Systems of Equations with Binary Dependent Variables," Econometrica, 79(3), 949-955.

Sherman, R. (1993): "The Limiting Distribution of the Maximum Rank Correlation Estimator," Econometrica, 61, 123-137. 
(1994a): "Maximal Inequalities for Degenerate U-Processes with Applications to Optimization Estimators," Annals of Statistics, 22, 439-459.

(1994b): "U-Processes in the Analysis of a Generalized Semiparametric Regression Estimator," Econometric Theory, 10, 372-395.

TAmer, E. (2003): "Incomplete Bivariate Discrete Response Model with Multiple Equilibria," Review of Economic Studies, 70(1), 147-167.

VuOng, Q., AND H. XU (2017): "Counterfactual mapping and individual treatment effects in nonseparable models with binary endogeneity," Quantitative Economics, 8(2), 589-610.

Vytlacil, E. J., And N. Yildiz (2007): "Dummy Endogenous Variables in Weakly Separable Models," Econometrica, 75(3), 757-779. 


\title{
Supplement to "Informational Content of Factor Structures in Simultaneous Binary Response Models"
}

\author{
Shakeeb Khan \\ Arnaud Maurel \\ Yichong Zhang \\ Boston College \\ Duke University, NBER and IZA \\ Singapore Management University
}

December 2020

\begin{abstract}
This paper gathers the supplementary material to the original paper. In Section E, we discuss the identification power of the factor structure. In Section F, we propose an estimator based on our constructive identification strategy and establish its asymptotic properties. Section G contains a simulation study. In Sections H and I, we prove Theorems 2.1 and 3.1, respectively. In Section J, we establish the asymptotic distribution for the rank estimator. In Section K, we consider the identification of the model with two idiosyncratic shocks but no continuous repeated measurements of the common factor. In Sections L, M, N and O, we prove Theorems E.1, E.2, K.1 and K.2, respectively.
\end{abstract}

Keywords: Factor Structures, Discrete Choice, Causal Effects.

\section{E Identification with and without Factor Structure}

\section{E.1 Identification Without Auxiliary Measurements}

In this section, we discuss the information content of factor structure. For illustration purpose, we focus on the "condensed" model:

$$
\begin{aligned}
& Y_{1}=\mathbf{1}\left\{X_{1}+\alpha_{0} Y_{2}-U \geq 0\right\} \\
& Y_{2}=\mathbf{1}\{X-V \geq 0\} .
\end{aligned}
$$




\section{Assumption 1.}

1. $\left(X_{1}, X\right) \perp(U, V)$.

2. $\left(X_{1}, X\right)$ are continuously distributed with absolute continuous joint density w.r.t. Lebesgue measure. The conditional support of $X_{1}$ given $X$ is $[a, b]$.

3. $V$ is continuously distributed over $\Re$ and its density w.r.t. Lebesgue measure exist.

Theorem E.1. If Assumption 1 holds, then $\left|\alpha_{0}\right| \leq b-a$ is necessary and sufficient for $\alpha_{0}$ to be identified.

We note that under Assumption 1, $\left|\alpha_{0}\right| \leq b-a$ is equivalent to the fact that we can find $x_{1}$ and $\tilde{x}_{1}$ in the support of $X_{1}$ such that $\alpha_{0}=x_{1}-\tilde{x}_{1}$.

Next, we assume, in addition to Assumption 1, the factor structure, i.e., (2.6) in Section 2. Our rank estimator can be written as an M-estimator

$$
\hat{\theta}=\arg \max _{\theta} Q_{n}(\theta) \equiv \sum_{i \neq j} \hat{g}_{i, j}(\theta)
$$

in which

$$
\begin{aligned}
\hat{g}_{i, j}(\theta) & =\left[\mathbf{1}\left\{\partial_{2} \hat{P}^{11}\left(X_{1, i}, X_{i}\right) / \hat{f}_{V}\left(X_{i}\right)+\partial_{2} \hat{P}^{10}\left(X_{1, j}, X_{j}\right) / \hat{f}_{V}\left(X_{j}\right) \geq 0\right\} \mathbf{1}\left\{\Phi\left(X_{1, i}, X_{i}, X_{1, j}, X_{j} ; \theta\right) \geq 0\right\}\right. \\
& \left.+\mathbf{1}\left\{\partial_{2} \hat{P}^{11}\left(X_{1, i}, X_{i}\right) / \hat{f}_{V}\left(X_{i}\right)+\partial_{2} \hat{P}^{10}\left(X_{1, j}, X_{j}\right) / \hat{f}_{V}\left(X_{j}\right)<0\right\} \mathbf{1}\left\{\Phi\left(X_{1, i}, X_{i}, X_{1, j}, X_{j} ; \theta\right)<0\right\}\right]
\end{aligned}
$$

with

$$
\Phi\left(x_{1}, x, \tilde{x}_{1}, \tilde{x} ; \theta\right)=x_{1}+\alpha-\gamma x-\left(\tilde{x}_{1}-\gamma \tilde{x}\right) .
$$

We will study the asymptotic properties of this estimator in Section F.

The information content explored by the M-estimator can be summarized as follows:

$$
\begin{aligned}
& \mathcal{A}_{2}(\theta)=\left\{\left(X_{1}, \tilde{X}_{1}, X, \tilde{X}\right), \Phi\left(X_{1}, X, \tilde{X}_{1}, \tilde{X} ; \theta_{0}\right) \geq 0>\Phi\left(X_{1}, X, \tilde{X}_{1}, \tilde{X} ; \theta\right)\right. \\
&\text { or } \left.\Phi\left(X_{1}, X, \tilde{X}_{1}, \tilde{X} ; \theta_{0}\right)<0 \leq \Phi\left(X_{1}, X, \tilde{X}_{1}, \tilde{X} ; \theta\right)\right\} .
\end{aligned}
$$

Then we cannot distinguish, from the true parameter $\theta_{0}$, all impostors in

$$
\overline{\mathcal{A}}_{2}=\left\{\theta: P\left(\mathcal{A}_{2}(\theta)\right)=0\right\}
$$

In the condensed model, if $\operatorname{Supp}\left(X_{1}, X\right)=[a, b] \times[c, d]$, then $\theta_{0}$ is identified if $\left|\alpha_{0}\right|<b-a+\left|\gamma_{0}\right|(d-c)$. Recall Theorem E.1, without imposing factor structure, the necessary and sufficient condition for achieving identification is $\left|\alpha_{0}\right| \leq b-a$. Therefore, the blue area in the Figure below is the additional parts of parameter space that are identified with factor structure but not otherwise. 


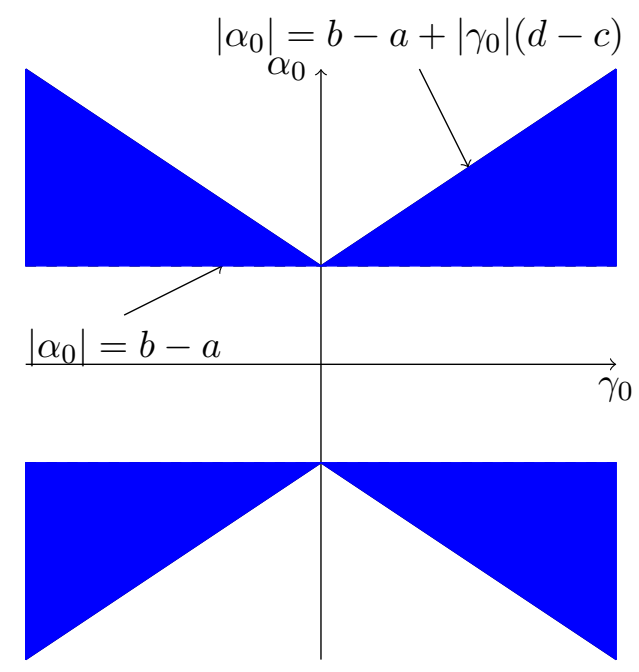

Figure 1: Identifying Power of Factor Structure

Theorem E.2. Assumption 1 holds. When $\left|\alpha_{0}\right|>b-a$, the sharp identified set for $\alpha_{0}$ is

$$
\mathcal{A}^{*}=\left\{\alpha: \alpha>b-a \text { if } \alpha_{0}>0 \text { and } \alpha<a-b \text { if } \alpha_{0}<0\right\} .
$$

Theorem E.2 highlights that, in the case without the factor structure and $\alpha_{0}$ does not satisfy the parameter restriction, except for the fact that the sign of $\alpha_{0}$ is identified, we actually cannot say much about the value of $\left|\alpha_{0}\right|$. When we assume the factor structure, the parameter is still not identified if $\left|\alpha_{0}\right|>b-a+\left|\gamma_{0}\right|(d-c)$. In addition, suppose $\alpha_{0}>0$. In this case, if we do not impose factor structure, by Theorem E.2, the sharp identified set is $\{\alpha: \alpha>b-a\}$ while with the factor structure, the identified set (not necessarily sharp) is $\alpha>b-a+|\gamma|(d-c)$. This implies, when identification fails in both cases, the blue area is also the extra identifying power on the identified set given by the factor structure.

\section{E.2 Identification with two auxiliary measurements}

Next, we expand our condensed model to include two continuous measurements. We show in this case, without the factor structure, $\alpha_{0}$ is not identified. This is in contrast with the identification result established in Theorem 3.1.

Suppose in addition to (E.4), we also observe two continuous measurements denoted as $Y_{3}$ and $Y_{4}$.

\section{Assumption 2.}

1. $\left(X_{1}, X\right) \perp\left(U, V, Y_{3}, Y_{4}\right)$.

2. $\left(X_{1}, X\right)$ are continuously distributed with absolute continuous joint density w.r.t. Lebesgue 
measure. The conditional support of $X_{1}$ given $X$ is $[a, b]$.

3. $V$ is continuously distributed over $\Re$ and its density w.r.t. Lebesgue measure exist.

Theorem E.3. If Assumption 2 holds, then $\left|\alpha_{0}\right| \leq b-a$ is necessary and sufficient for $\alpha_{0}$ to be identified.

The proof of Theorem E.3 is similar to that of Theorem E.1, and thus, is omitted. In the proof of Theorem E.1, we show that when $\left|\alpha_{0}\right|>b-a$, we can find an impostor $\alpha \neq \alpha_{0}$ and $\tilde{U}$ such that for any $x_{1} \in[a, b]$ and any $v \in \operatorname{Supp}(V)$, we have

$$
\begin{aligned}
& P\left(\tilde{U} \leq x_{1}+\alpha \mid V=v\right)=P\left(U \leq x_{1}+\alpha_{0} \mid V=v\right) \\
& P\left(\tilde{U} \leq x_{1} \mid V=v\right)=P\left(U \leq x_{1} \mid V=v\right) .
\end{aligned}
$$

This implies the conditional CDF of $\left(Y_{1}, Y_{2}\right)$ given $\left(X_{1}, X\right)$ under the DGPs $\left(U, V, \alpha_{0}\right)$ and $(\tilde{U}, V, \alpha)$ are the same, and thus, $\alpha_{0}$ is observationally equivalent to the impostor $\alpha$. Similarly, with the two continuous measurements, we can use the exact same construction of $\tilde{U}$ and $\alpha$ to show that, for any $x_{1} \in[a, b]$ and $\left(v, y_{3}, y_{4}\right) \in \operatorname{Supp}\left(V, Y_{3}, Y_{4}\right)$, we have

$$
\begin{aligned}
& P\left(\tilde{U} \leq x_{1}+\alpha \mid V=v, Y_{3}=y_{3}, Y_{4}=y_{4}\right)=P\left(U \leq x_{1}+\alpha_{0} \mid V=v, Y_{3}=y_{3}, Y_{4}=y_{4}\right) \\
& P\left(\tilde{U} \leq x_{1} \mid V=v, Y_{3}=y_{3}, Y_{4}=y_{4}\right)=P\left(U \leq x_{1} \mid V=v, Y_{3}=y_{3}, Y_{4}=y_{4}\right) .
\end{aligned}
$$

This implies the conditional CDF of $\left(Y_{1}, Y_{2}, Y_{3}, Y_{4}\right)$ given $\left(X_{1}, X\right)$ under the DGPs $\left(U, V, Y_{3}, Y_{4}, \alpha_{0}\right)$ and $\left(\tilde{U}, V, Y_{3}, Y_{4}, \alpha\right)$ are the same too. Such non-identification result holds even when $X$ has full support.

\section{F Estimation and Asymptotic Properties}

Our identification result is constructive in the sense that it motivates an estimator for the parameters of interest which we describe in detail here.

As we did in Section E, to simplify exposition, in the following we focus exclusively on the parameters $\alpha_{0}, \gamma_{0}$. Recall the choice probabilities $P^{i j}\left(x_{1}, x\right)=\operatorname{Prob}\left(Y_{1}=i, Y_{2}=j \mid X_{1}=x_{1}, X=x\right)$ and its second derivative $\partial_{2} P^{i j}\left(x_{1}, x\right)$, which can be estimated as we describe below. Another function needed for our identification result is the density function of the unobserved term $V$, denoted by $f_{V}(\cdot)$. This is also unknown, but from the structure of our model can be recovered from the derivative with respect to the instrument $X$ of $E\left[Y_{2} \mid X\right]$, and hence is estimable from the data. Note that the proof of Theorem 2.1 shows that the sign of the index evaluated at two different regressor values, which we denote here by $\left(X_{1}, X\right)$ and $\left(\tilde{X}_{1}, \tilde{X}\right)$ is determined by the choice probabilities via

$$
\partial_{2} P^{11}\left(X_{1}, X\right) / f_{V}(X)+\partial_{2} P^{10}\left(\tilde{X}_{1}, \tilde{X}\right) / f_{V}(\tilde{X}) \geq 0 \quad \Longleftrightarrow X_{1}+\alpha-\gamma X-\left(\tilde{X}_{1}-\gamma \tilde{X}\right) \geq 0 .
$$


This motivates us to use the maximum rank correlation estimator proposed by Han (1987).

Implementation requires further details to pay attention to. The unknown choice probabilities, their derivatives, and the density of $V$ will be estimated using nonparametric methods, and for this we adopt locally linear methods as they are particularly well suited for estimating derivatives of functions.

With functions and their derivatives estimated in the first stage of our procedure, the second stage plugs in these estimated values into an objective function to be optimized. Specifically, letting $\hat{\theta}$ denote $(\hat{\alpha}, \hat{\gamma})$, our estimator is of the form:

$$
\hat{\theta}=\arg \max _{\theta} Q_{n}(\theta), \quad Q_{n}(\theta) \equiv \sum_{i \neq j} \hat{g}_{i, j}(\theta)
$$

in which

$$
\begin{aligned}
\hat{g}_{i, j}(\theta) & =\left[\mathbf{1}\left\{\partial_{2} \hat{P}^{11}\left(X_{1, i}, X_{i}\right) / \hat{f}_{V}\left(X_{i}\right)+\partial_{2} \hat{P}^{10}\left(X_{1, j}, X_{j}\right) / \hat{f}_{V}\left(X_{j}\right) \geq 0\right\} \mathbf{1}\left\{\Phi\left(X_{1, i}, X_{i}, X_{1, j}, X_{j} ; \theta\right) \geq 0\right\}\right. \\
& \left.+\mathbf{1}\left\{\partial_{2} \hat{P}^{11}\left(X_{1, i}, X_{i}\right) / \hat{f}_{V}\left(X_{i}\right)+\partial_{2} \hat{P}^{10}\left(X_{1, j}, X_{j}\right) / \hat{f}_{V}\left(X_{j}\right)<0\right\} \mathbf{1}\left\{\Phi\left(X_{1, i}, X_{i}, X_{1, j}, X_{j} ; \theta\right)<0\right\}\right],
\end{aligned}
$$

with

$$
\Phi\left(x_{1}, x, \tilde{x}_{1}, \tilde{x} ; \theta\right)=x_{1}+\alpha-\gamma x-\left(\tilde{x}_{1}-\gamma \tilde{x}\right) .
$$

We note that this estimator falls into the class of those which optimize a nonsmooth U-process involving components estimated nonparametrically in a preliminary stage. ${ }^{1}$ Examples of other estimators in this class can be found in Khan (2001), Abrevaya, Hausman, and Khan (2010), Jochmans (2013), Chen, Khan, and Tang (2016), and our approach to deriving the limiting distribution theory of our estimator will follow along the steps used in those papers. Our limiting distribution theory for this estimator is based on the following regularity conditions:

RK1 $\theta_{0}$ lies in the interior of $\Theta$, a compact subset of $R^{2}$.

RK2 The index $X$ is continuously distributed with support on the real line, and has a density function which is twice continuously differentiable.

RK3 (Order of smoothness of probability functions and regressor density functions) The functions $P^{i, j}(\cdot)$ and $f_{X_{1}, X}(\cdot \cdot)$ (the density function of the random vector $\left(X_{1}, X\right)$ ) are continuously

\footnotetext{
${ }^{1}$ An alternative estimation procedure could be based on the exact relationship in (2.7). Note the equality on the left-hand side of (2.7) is a function of the data alone and not the unknown parameters. The right-hand side equality can then be regarded as a moment condition to estimate the unknown parameters. We describe this estimator and derive its asymptotic properties in the Online Supplement to the paper. While the two estimation approaches will have similar asymptotic properties (root- $n$ consistent, asymptotically normal), we prefer the rank estimator in (F.5) which involves fewer tuning parameters. Furthermore rank type estimators in general are more robust to certain types of misspecification, as pointed out in Khan and Tamer (2018).
} 
differentiable of order $p_{2}$.

RK4 (First stage kernel function conditions) $K(\cdot)$, used to estimate the choice probabilities and their derivatives is an even function, integrating to 1 and is of order $p_{2}$.

RK5 (Rate condition on first stage bandwidth sequence) The first stage bandwidth sequence $H_{n}$ used in the nonparametric estimator of the choice probability functions and their derivatives satisfies $\sqrt{n} H_{n}^{p_{2}-1} \rightarrow 0$ and $n^{-1 / 4} H_{n}^{-1} \rightarrow 0$.

The smoothness condition in Assumption RK4 and Assumption RK5 is due to the fact that we need to nonparametrically estimate $\partial_{2} P^{i j}\left(X_{1}, X\right)$ with sufficiently faster convergence rate. This will require a stronger smoothness condition than that required for standard nonparametric estimation. Assumption RK5 ensures that the bias of the first stage estimator of the derivative function converges at the parametric rate and the RMSE of this estimator (with two regressors) is fourth-root consistent, so results for two step estimation in Newey and McFadden (1994) can be applied.

Based on these conditions, we have the following theorem, whose proof is in Section $\mathrm{J}$ of the Supplementary Appendix which characterizes the rate of convergence and asymptotic distribution of the proposed estimator:

Theorem F.1. Under Assumptions RK1-RK5,

$$
\sqrt{n}\left(\hat{\theta}-\theta_{0}\right) \Rightarrow N\left(0, V^{-1} \Delta V^{-1}\right)
$$

where the forms of the Hessian term $V$ and outer score term $\Delta$ are described in detail in Section $J$ of the Supplementary Appendix.

\section{G Finite Sample Properties}

In this section we explore the finite sample properties of the proposed estimation procedure via a simulation study. We will also see how sensitive the performance of the proposed estimator is to the factor structure assumption. As a base comparison, we also report results for the estimator proposed in Vytlacil and Yildiz (2007) to see how sensitive it is to their second instrument restriction.

Our data are simulated from base models of the form

$$
\begin{aligned}
& Y_{1}=\mathbf{1}\left\{X_{1}+\alpha_{0} Y_{2}-U \geq 0\right\} \\
& Y_{2}=\mathbf{1}\{X-V>0\}
\end{aligned}
$$

where $X_{1}$ is binary with success probability $0.6, X$ has marginal distribution $\mathcal{N}(0,1), X_{1}$ and $X$ are mutually independent, $\left(X_{1}, X\right) \perp(V, \Pi), U=\gamma_{0} V+\Pi$. $(V, \Pi)$ are distributed independently 
of each other, where $\Pi$ is distributed following a standard normal distribution, and $V$ is distributed either standard normal, Laplace, or $T(3)$. The parameters $\left(\alpha_{0}, \gamma_{0}\right)=(-0.25,1.2)$ or $(0.5,1.2)$.

Since $X_{1}$ is discrete, Vytlacil and Yildiz's (2007) identification condition does not hold. However, the identification condition in this paper becomes

$$
|\alpha| \leq \text { length of the support of } X
$$

which holds.

For each choice of sample size $n=100,200,400,800,1,600$, we simulate 280 samples and report the bias, standard deviation (std), root mean squared error (RMSE), and median absolute deviation (MAD) for both Vytlacil and Yildiz's (2007) estimator (VY) and ours (KMZ). For implementation, we use the second order local polynomial along with Gaussian kernels to nonparametrically estimate the $\partial_{2} P^{11}\left(x_{1}, x\right)$ and $\partial_{2} P^{10}\left(x_{1}, x\right)$. The bandwidth we use is $h_{1}=\sigma_{x} N^{-1 / 7}$ where $\sigma_{x}$ is the standard deviation of $X . f_{V}(x)$ is nonparametrically estimated using a local linear estimator with the tuning parameter $h_{2}=\sigma_{x} N^{-1 / 6}$.

As results from the table indicate, the finite sample performance of our estimator generally agrees with the asymptotic theory. The RMSE for the estimator proposed here is decreasing as the sample size increases, as one could expect given the consistency property of our estimator. Besides, the decay rate of the RMSE and MAD is about $\sqrt{2}$ when $n \geq 400$ as sample sizes doubles, in line with the parametric rate of convergence of our estimator.

Vytlacil and Yildiz's (2007) estimator, which does not exploit the factor structure, demonstrates inconsistency for certain parameter values, as indicated by the bias and median bias not shrinking with the sample size. In addition, the RMSE and MAD do not appear to decline at all, which also suggests that Vytlacil and Yildiz's (2007) estimator is inconsistent in these designs. ${ }^{2}$

Table 1: Normal $V, \alpha=0.5$

\begin{tabular}{|c|c|c|c|c|c|c|c|c|c|c|c|c|c|c|c|c|c|c|}
\hline \multirow[t]{2}{*}{$\Pi$} & \multicolumn{6}{|c|}{ Normal } & \multicolumn{6}{|c|}{ Laplace } & \multicolumn{6}{|c|}{$\mathrm{T}(3)$} \\
\hline & \multicolumn{3}{|c|}{$\mathrm{kmz}$} & \multicolumn{3}{|c|}{ vy } & \multicolumn{3}{|c|}{$\mathrm{kmz}$} & \multicolumn{3}{|c|}{ vy } & \multicolumn{3}{|c|}{$\mathrm{kmz}$} & \multicolumn{3}{|c|}{ vy } \\
\hline $\mathrm{N}$ & Bias & RMSE & MAD & Bias & RMSE & MAD & Bias & RMSE & MAD & Bias & RMSE & MAD & Bias & RMSE & MAD & Bias & RMSE & MAD \\
\hline 100 & -0.026 & 0.665 & 0.660 & -0.246 & 0.658 & 0.500 & 0.032 & 0.634 & 0.560 & -0.293 & 0.658 & 0.500 & 0.010 & 0.676 & 0.665 & -0.225 & 0.662 & 0.500 \\
\hline 200 & 0.004 & 0.591 & 0.475 & -0.329 & 0.633 & 0.500 & -0.015 & 0.568 & 0.400 & -0.336 & 0.612 & 0.500 & -0.003 & 0.616 & 0.495 & -0.279 & 0.629 & 0.500 \\
\hline 400 & 0.005 & 0.483 & 0.365 & -0.341 & 0.573 & 0.500 & 0.030 & 0.459 & 0.310 & -0.323 & 0.559 & 0.500 & 0.018 & 0.542 & 0.405 & -0.314 & 0.589 & 0.500 \\
\hline 800 & 0.065 & 0.456 & 0.300 & -0.348 & 0.544 & 0.500 & 0.096 & 0.391 & 0.250 & -0.357 & 0.511 & 0.500 & 0.046 & 0.462 & 0.295 & -0.346 & 0.552 & 0.500 \\
\hline 1,600 & 0.040 & 0.321 & 0.195 & -0.413 & 0.503 & 0.500 & 0.017 & 0.294 & 0.190 & -0.450 & 0.506 & 0.500 & 0.034 & 0.371 & 0.240 & -0.368 & 0.506 & 0.500 \\
\hline
\end{tabular}

\footnotetext{
${ }^{2}$ Because $X_{1}$ is binary, Vytlacil and Yildiz's (2007) estimator can only take 3 possible values: $0,-1$ or 1 . In particular, when $\alpha=0.5$, in most of the replications, the estimator takes values 0 or 1 . When $\alpha=-0.25$, in most of the replications, the estimator takes value -1 . In both of these cases, the MAD remains constant over the different sample sizes.
} 
Table 2: Normal $V, \alpha=-0.25$

\begin{tabular}{|c|c|c|c|c|c|c|c|c|c|c|c|c|c|c|c|c|c|c|}
\hline \multirow[t]{2}{*}{$\Pi$} & \multicolumn{6}{|c|}{ Normal } & \multicolumn{6}{|c|}{ Laplace } & \multicolumn{6}{|c|}{$\mathrm{T}(3)$} \\
\hline & \multicolumn{3}{|c|}{$\mathrm{kmz}$} & \multicolumn{3}{|c|}{ vy } & \multicolumn{3}{|c|}{$\mathrm{kmz}$} & \multicolumn{3}{|c|}{ vy } & \multicolumn{3}{|c|}{$\mathrm{kmz}$} & \multicolumn{3}{|c|}{ vy } \\
\hline $\mathrm{N}$ & Bias & RMSE & MAD & Bias & RMSE & MAD & Bias & RMSE & MAD & Bias & RMSE & MAD & Bias & RMSE & MAD & Bias & RMSE & MAD \\
\hline 100 & -0.088 & 0.650 & 0.555 & -0.466 & 0.710 & 0.750 & 0.092 & 0.614 & 0.530 & -0.358 & 0.650 & 0.750 & 0.004 & 0.619 & 0.505 & -0.430 & 0.681 & 0.750 \\
\hline 200 & -0.035 & 0.599 & 0.420 & -0.446 & 0.681 & 0.750 & 0.012 & 0.552 & 0.385 & -0.485 & 0.689 & 0.750 & -0.008 & 0.583 & 0.425 & -0.463 & 0.687 & 0.750 \\
\hline 400 & -0.016 & 0.467 & 0.325 & -0.487 & 0.668 & 0.750 & -0.010 & 0.388 & 0.200 & -0.552 & 0.686 & 0.750 & -0.003 & 0.496 & 0.340 & -0.489 & 0.675 & 0.750 \\
\hline 800 & -0.028 & 0.324 & 0.165 & -0.591 & 0.697 & 0.750 & 0.006 & 0.279 & 0.180 & -0.599 & 0.701 & 0.750 & 0.032 & 0.399 & 0.230 & -0.533 & 0.682 & 0.750 \\
\hline 1,600 & -0.006 & 0.244 & 0.150 & -0.654 & 0.718 & 0.750 & -0.028 & 0.204 & 0.130 & -0.714 & 0.738 & 0.750 & -0.021 & 0.279 & 0.190 & -0.629 & 0.710 & 0.750 \\
\hline
\end{tabular}

In the following, we also consider three DGPs (DGPs 1-3) such that the one-factor model does not hold but the identification assumption in Vytlacil and Yildiz (2007) does. In this case, our simulation results show that while, as expected, the estimator VY is still valid, our estimator still performs reasonably well. Interestingly, this offers suggestive evidence that our estimator is robust to some degree of misspecification. As such, these results complement previous work highlighting the robustness of rank type estimators to misspecification Khan and Tamer (2018). In DGP 4, the identification assumptions in both Vytlacil and Yildiz (2007) and our paper hold. In this case, we found that our estimator has similar performance as that proposed by Vytlacil and Yildiz (2007).

The outcome and selection equations are the same as (G.1) and (G.2), respectively. Then,

DGP $1:\left(X_{1}, X\right)$ is jointly standard normally distributed. Let $\left(e_{1}, e_{2}\right)$ jointly Laplace distributed with mean zero and variance-covariance matrix $\Sigma=\left(\begin{array}{cc}1 & -0.5 \\ -0.5 & 1\end{array}\right), e_{3}$ and $e_{4}$ are uniformly distributed on $(0,1)$, independent of each other, and independent of $\left(e_{1}, e_{2}\right), V=e_{1}+e_{3}-0.5$, $U=e_{2}+e_{4}-0.5$, and $\alpha=-0.25$.

DGP $2:\left(X_{1}, X\right)$ are the same as above, $U=e_{1}+e_{2}-0.5$, and $V=e_{1}+e_{3}-0.5$, where $e_{1}$ is standard normally distributed, $\left(e_{2}, e_{3}\right)$ are uniformly distributed on $(0,1),\left(e_{1}, e_{2}, e_{3}\right)$ are mutually independent, and $\alpha=-0.25$.

DGP $3:\left(X_{1}, X\right)$ are the same as above, $V=\frac{\exp \left(e_{1}+e_{2}-0.5\right)-1}{4}, U=\frac{\exp \left(e_{1}+e_{3}-0.5\right)-1}{4},\left(e_{1}, e_{2}, e_{3}\right)$ are defined as above, and $\alpha=-0.5$.

DGP $4:\left(X_{1}, X\right)$ are the same as above, $V$ is Laplace distributed with mean zero and standard derivation $0.5, U=V+V^{\prime}-0.5$, where $V^{\prime}$ is uniform distributed on $(0,1)$ and is independent of $V$, and $\alpha=-0.25$.

For DGPs 1, 2, and 4, when computing $\partial_{2} P^{11}\left(x_{1}, x\right)$ and $\partial_{2} P^{10}\left(x_{1}, x\right)$, we use bandwidths $h_{1}=$ $\sigma_{x 1} N^{-1 / 7}$ and $h=\sigma_{x} N^{-1 / 7}$ for variables $X_{1}$ and $X$, respectively, where $\sigma_{x 1}$ and $\sigma_{x}$ are the standard errors of $X_{1}$ and $X$, respectively. To estimate the density $f_{V}(x)$, we use bandwidth $h_{2}=\sigma_{x} N^{-1 / 6}$. For DGP 3, we use $h_{1}=h_{2}=h=\sigma_{x 1} N^{-1 / 5}$. In all simulations, we use 280 replications. 
Table 3: Alternative DGPs

\begin{tabular}{|c|c|c|c|c|c|c|c|c|c|c|c|c|}
\hline & \multicolumn{6}{|c|}{ DGP 1} & \multicolumn{6}{|c|}{ DGP 2} \\
\hline & \multicolumn{3}{|c|}{$\mathrm{kmz}$} & \multicolumn{3}{|c|}{ vy } & \multicolumn{3}{|c|}{$\mathrm{kmz}$} & \multicolumn{3}{|c|}{ vy } \\
\hline $\mathrm{N}$ & Bias & RMSE & MAD & Bias & RMSE & MAD & Bias & RMSE & MAD & Bias & RMSE & MAD \\
\hline 100 & -0.065 & 0.678 & 0.600 & -0.055 & 0.666 & 0.535 & -0.058 & 0.621 & 0.505 & -0.05 & 0.621 & 0.470 \\
\hline 200 & -0.118 & 0.543 & 0.370 & -0.080 & 0.497 & 0.320 & -0.122 & 0.523 & 0.350 & -0.097 & 0.495 & 0.350 \\
\hline 400 & -0.117 & 0.413 & 0.280 & -0.071 & 0.378 & 0.245 & -0.062 & 0.335 & 0.215 & -0.033 & 0.316 & 0.220 \\
\hline 800 & -0.102 & 0.287 & 0.170 & -0.062 & 0.243 & 0.160 & -0.031 & 0.242 & 0.150 & -0.008 & 0.215 & 0.150 \\
\hline \multirow[t]{2}{*}{1,600} & -0.071 & 0.193 & 0.140 & -0.035 & 0.155 & 0.100 & -0.038 & 0.167 & 0.100 & -0.031 & 0.158 & 0.100 \\
\hline & \multicolumn{6}{|c|}{ DGP 3} & \multicolumn{6}{|c|}{ DGP 4} \\
\hline 100 & -0.012 & 0.583 & 0.480 & -0.015 & 0.565 & 0.430 & -0.057 & 0.401 & 0.240 & -0.066 & 0.422 & 0.240 \\
\hline 200 & -0.061 & 0.425 & 0.275 & -0.068 & 0.399 & 0.270 & -0.041 & 0.282 & 0.180 & -0.049 & 0.263 & 0.145 \\
\hline 400 & -0.041 & 0.259 & 0.170 & -0.042 & 0.237 & 0.155 & -0.062 & 0.184 & 0.135 & -0.047 & 0.186 & 0.120 \\
\hline 800 & -0.061 & 0.219 & 0.140 & -0.047 & 0.182 & 0.120 & -0.029 & 0.119 & 0.080 & -0.034 & 0.115 & 0.070 \\
\hline 1,600 & -0.038 & 0.130 & 0.080 & -0.035 & 0.119 & 0.080 & -0.024 & 0.090 & 0.060 & -0.022 & 0.086 & 0.070 \\
\hline
\end{tabular}

In the first three DGPs, we see that VY's estimator has better performance in terms of both bias and MSE. On the other hand, although the models do not have a factor structure, our estimator still performs reasonably well. In the last DGP, support conditions in both Vytlacil and Yildiz (2007) and our paper hold. Table 3 shows that our and Vytlacil and Yildiz's (2007) estimators have similar performance in terms of bias and MSE. Although our estimator is expected to be more efficient as we use the factor structure in estimation, it is not. We conjecture that it is because our estimator does not necessarily use all the information, or in other words, achieve the semiparametric efficiency bound. To establish the semiparametric efficient estimator in the model with and without the factor structure is an interesting yet challenging task. We leave it as a topic for future research.

\section{H Proof of Theorem 2.1}

Proof: Note that

$$
\begin{aligned}
& P^{11}\left(z_{1}, z_{3}, x\right)=\int_{-\infty}^{x} F_{\Pi}\left(z_{1}^{\prime} \lambda_{0}+z_{3}^{\prime} \beta_{0}+\alpha_{0}-\gamma_{0} v\right) f_{V}(v) d v \\
& P^{10}\left(\tilde{z}_{1}, \tilde{z}_{3}, \tilde{x}\right)=\int_{\tilde{x}}^{+\infty} F_{\Pi}\left(\tilde{z}_{1}^{\prime} \lambda_{0}+\tilde{z}_{3}^{\prime} \beta_{0}-\gamma_{0} v\right) f_{V}(v) d v .
\end{aligned}
$$

Taking derivatives w.r.t. the third argument of the LHS function, we obtain

$$
\begin{aligned}
\partial_{x} P^{11}\left(z_{1}, z_{3}, x\right) / f_{V}(x) & =F_{\Pi}\left(z_{1}^{\prime} \lambda_{0}+z_{3}^{\prime} \beta_{0}+\alpha_{0}-\gamma_{0} x\right) \\
-\partial_{x} P^{10}\left(\tilde{z}_{1}, \tilde{z}_{3}, \tilde{x}\right) / f_{V}(\tilde{x}) & =F_{\Pi}\left(\tilde{z}_{1}^{\prime} \lambda_{0}+\tilde{z}_{3}^{\prime} \beta_{0}-\gamma_{0} \tilde{x}\right) .
\end{aligned}
$$

By Assumption A4, we know that there exists pairs such that

$$
Z_{1}^{\prime} \lambda_{0}+Z_{3}^{\prime} \beta_{0}+\alpha_{0}-\gamma_{0} X=\tilde{Z}_{1}^{\prime} \lambda_{0}+\tilde{Z}_{3}^{\prime} \beta_{0}-\gamma_{0} \tilde{X}
$$


Because $F_{\Pi}(\cdot)$ is monotone increasing, we have

$$
\begin{aligned}
& \partial_{x} P^{11}\left(Z_{1}, Z_{3}, X\right) / f_{V}(X)+\partial_{x} P^{10}\left(\tilde{Z}_{1}, \tilde{Z}_{3}, \tilde{X}\right) / f_{V}(\tilde{X})=0 \\
\Longleftrightarrow & \alpha_{0}+\left(Z_{1}-\tilde{Z}_{1}\right)^{\prime} \lambda_{0}+\left(Z_{3}-\tilde{Z}_{3}\right)^{\prime} \beta_{0}-\gamma_{0}(X-\tilde{X})=0
\end{aligned}
$$

Note the LHS of the above display is identified from data. Denote $Z_{1,1}$ as the first element of $Z_{1}$, whose coefficient is set to one. The rest of $Z_{1}$ is denoted as $Z_{1,-1}$, whose coefficient is denoted as $\lambda_{0,-1}$. Then, we have

$$
\alpha_{0}+\left(Z_{1,-1}-\tilde{Z}_{1,-1}\right)^{\prime} \lambda_{0,-1}+\left(Z_{3}-\tilde{Z}_{3}\right)^{\prime} \beta_{0}-\gamma_{0}(X-\tilde{X})=\tilde{Z}_{1,1}-Z_{1,1} .
$$

Then, by Assumption A4, we can find $\left(z_{1}^{(l)}, z_{3}^{(l)}, x^{(l)}\right)_{l=1}^{d}$ and $\left(\tilde{z}_{1}^{(l)}, \tilde{z}_{3}^{(l)}, \tilde{x}^{(l)}\right)_{l=1}^{d}$ such that

$$
\operatorname{rank}\left(\begin{array}{ccc}
1 & \cdots & 1 \\
z_{1,-1}^{(1)}-\tilde{z}_{1,-1}^{(1)} & \cdots & z_{1,-1}^{(d)}-\tilde{z}_{1,-1}^{(d)} \\
z_{3}^{(1)}-\tilde{z}_{3}^{(1)} & \cdots & z_{3}^{(d)}-\tilde{z}_{3}^{(d)} \\
x^{(1)}-\tilde{x}^{(1)} & \cdots & x^{(d)}-\tilde{x}^{(d)}
\end{array}\right)=d .
$$

Then, we can identify $\left(\alpha_{0}, \lambda_{0}, \beta_{0}, \gamma_{0}\right)$ by solving the linear system that

$$
\begin{gathered}
\alpha_{0}+\left(z_{1,-1}^{(1)}-\tilde{z}_{1,-1}^{(1)}\right)^{\prime} \lambda_{0,-1}+\left(z_{3}^{(1)}-\tilde{z}_{3}^{(1)}\right)^{\prime} \beta_{0}-\gamma_{0}\left(x^{(1)}-\tilde{x}^{(1)}\right)=\tilde{z}_{1,1}^{(1)}-z_{1,1}^{(1)}, \\
\vdots \\
\alpha_{0}+\left(z_{1,-1}^{(d)}-\tilde{z}_{1,-1}^{(d)}\right)^{\prime} \lambda_{0,-1}+\left(z_{3}^{(d)}-\tilde{z}_{3}^{(d)}\right)^{\prime} \beta_{0}-\gamma_{0}\left(x^{(d)}-\tilde{x}^{(d)}\right)=\tilde{z}_{1,1}^{(d)}-z_{1,1}^{(d)} .
\end{gathered}
$$

This concludes the proof.

\section{Proof of Theorem 3.1}

For notation simplicity, we write $\tilde{W}=\nu_{0} W, \tilde{\sigma}_{0}=\sigma_{0} / \nu_{0}, \tilde{\nu}_{0}=1 / \nu_{0}$, and

$$
\begin{aligned}
& Y_{2}=\mathbf{1}\left\{X \geq \tilde{\nu}_{0} \tilde{W}+\eta_{2}\right\} \\
& Y_{3}=\tilde{W}+\eta_{3} \\
& Y_{4}=\tilde{\sigma}_{0} \tilde{W}+\eta_{4} .
\end{aligned}
$$

Because Assumptions B2-B6 hold, by applying Hu and Schennach (2013, Theorem 1) to $Y_{3}$ and $Y_{4}$, we can identify the densities for $\nu_{0} W=\tilde{W}, \eta_{3}$, and $\eta_{4}$ as well as $\sigma_{0} / \nu_{0}=\tilde{\sigma}_{0}$. 
Then, we have

$$
\begin{aligned}
\partial_{y_{3}} \mathbb{P}\left(Y_{2}=1, Y_{3} \leq y_{3} \mid X=x\right) & =\partial_{y_{3}} \int F_{\eta_{2}}\left(x-\tilde{\nu}_{0} w\right) F_{\eta_{3}}\left(y_{3}-w\right) f_{\tilde{W}}(w) d w \\
& =\int F_{\eta_{2}}\left(x-\tilde{\nu}_{0} w\right) f_{\eta_{3}}\left(y_{3}-w\right) f_{\tilde{W}}(w) d w
\end{aligned}
$$

Applying Fourier transform w.r.t. $y_{3}$ on both sides, we have

$$
\mathcal{F}\left(\partial_{y_{3}} \mathbb{P}\left(Y_{2}=1, Y_{3} \leq \cdot \mid X=x\right)\right)(t)=\mathcal{F}\left(F_{\eta_{2}}\left(x-\tilde{\nu}_{0} \cdot\right) f_{\tilde{W}}(\cdot)\right)(t) \mathcal{F}\left(f_{\eta_{3}}(\cdot)\right)(t)
$$

where for a generic function $g(w)$,

$$
\mathcal{F}(g(\cdot))(t)=\frac{1}{\sqrt{2 \pi}} \int \exp (-2 \pi i t w) g(w) d w
$$

Therefore,

$$
\frac{\mathcal{F}^{-1}\left(\frac{\mathcal{F}\left(\partial_{y_{3}} \mathbb{P}\left(Y_{2}=1, Y_{3} \leq \cdot \mid X=x\right)\right)(\cdot)}{\mathcal{F}\left(f_{\eta_{3}}(\cdot)\right)(\cdot)}\right)(w)}{f_{\tilde{W}}(w)}=F_{\eta_{2}}\left(x-\tilde{\nu}_{0} w\right)
$$

where for a generic function $g(w)$,

$$
\mathcal{F}^{-1}(g(\cdot))(t)=\frac{1}{\sqrt{2 \pi}} \int \exp (2 \pi i t w) g(w) d w
$$

Note the LHS of (I.1) can be identified from data. We choose two pairs $(x, w)$ and $\left(x^{\prime}, w^{\prime}\right)$ such that $w \neq w^{\prime}$ and

$$
\frac{\mathcal{F}^{-1}\left(\frac{\mathcal{F}\left(\partial_{y_{3}} \mathbb{P}\left(Y_{2}=1, Y_{3} \leq \cdot \mid X=x\right)\right)(\cdot)}{\mathcal{F}\left(f_{\eta_{3}}(\cdot)\right)(\cdot)}\right)(w)}{f_{\tilde{W}}(w)}=\frac{\mathcal{F}^{-1}\left(\frac{\mathcal{F}\left(\partial_{y_{3}} \mathbb{P}\left(Y_{2}=1, Y_{3} \leq \cdot \mid X=x^{\prime}\right)\right)(\cdot)}{\mathcal{F}\left(f_{\eta_{3}}(\cdot)\right)(\cdot)}\right)\left(w^{\prime}\right)}{f_{\tilde{W}}\left(w^{\prime}\right)} .
$$

Then, given the monotonicity of $F_{\eta_{2}}$, we have

$$
x-\tilde{\nu}_{0} w=x^{\prime}-\tilde{\nu}_{0} w^{\prime}
$$

or

$$
\tilde{\nu}_{0}=\left(x-x^{\prime}\right) /\left(w-w^{\prime}\right)
$$

which is identified. Given the identification of $\tilde{\nu}_{0}$ and the distribution of $\tilde{W}$, we can identify the distribution of $W=\tilde{\nu}_{0} \tilde{W}$. Recall $F_{\eta_{1}}(\cdot)$ and $f_{\eta_{2}}(\cdot)$ are the CDF and PDF of $\eta_{1}$ and $\eta_{2}$, respectively. Then, we have

$$
P\left(Y_{2}=1 \mid X=x\right)=P\left(W+\eta_{2} \leq x\right)
$$


Because $X$ has full support, we can identify the distribution of $W+\eta_{2}$. Then, it follows from standard deconvolution argument and the fact that the distribution of $W$ is identified that we can identify the distribution of $\eta_{2}$. In addition, note that

$$
\begin{aligned}
P^{11}\left(z_{1}, z_{3}, x\right) & =P\left(Y_{1}=1, Y_{2}=1 \mid Z_{1}=z_{1}, Z_{3}=z_{3}, X=x\right) \\
& =\int F_{\eta_{1}}\left(z_{1}^{\prime} \lambda_{0}+z_{3}^{\prime} \beta_{0}+\alpha_{0}-\gamma_{0} w\right) F_{\eta_{2}}(x-w) f_{W}(w) d w
\end{aligned}
$$

and

$$
\begin{aligned}
P^{10}\left(z_{1}, z_{3}, x\right) & =P\left(Y_{1}=1, Y_{2}=0 \mid Z_{1}=z_{1}, Z_{3}=z_{3}, X=x\right) \\
& =\int F_{\eta_{1}}\left(z_{1}^{\prime} \lambda_{0}+z_{3}^{\prime} \beta_{0}-\gamma_{0} w\right)\left(1-F_{\eta_{2}}(x-w)\right) f_{W}(w) d w .
\end{aligned}
$$

Taking derivatives of $P^{11}\left(z_{1}, z_{3}, x\right)$ and $P^{10}\left(z_{1}, z_{3}, x\right)$ w.r.t. $x$, we have

$$
\partial_{x} P^{11}\left(z_{1}, z_{3}, x\right)=\int F_{\eta_{1}}\left(z_{1}^{\prime} \lambda_{0}+z_{3}^{\prime} \beta_{0}+\alpha_{0}-w\right) f_{\eta_{2}}(x-w) f_{W}(w) d w
$$

and

$$
-\partial_{x} P^{10}\left(z_{1}, z_{3}, x\right)=\int F_{\eta_{1}}\left(z_{1}^{\prime} \lambda_{0}+z_{3}^{\prime} \beta_{0}-\gamma_{0} w\right) f_{\eta_{2}}(x-w) f_{W}(w) d w
$$

Applying Fourier transform on both sides of (I.2) and (I.3), we have

$$
\mathcal{F}\left(\partial_{x} P^{11}\left(z_{1}, z_{3}, \cdot\right)\right)=\mathcal{F}\left(F_{\eta_{1}}\left(z_{1}^{\prime} \lambda_{0}+z_{3}^{\prime} \beta_{0}+\alpha_{0}-\cdot\right) f_{W}(\cdot)\right) \mathcal{F}\left(f_{\eta_{2}}(\cdot)\right)
$$

and

$$
\mathcal{F}\left(-\partial_{x} P^{10}\left(z_{1}, z_{3}, \cdot\right)\right)=\mathcal{F}\left(F_{\eta_{1}}\left(z_{1}^{\prime} \lambda_{0}+z_{3}^{\prime} \beta_{0}-\cdot\right) f_{W}(\cdot)\right) \mathcal{F}\left(f_{\eta_{2}}(\cdot)\right)
$$

Then, by (I.4), we can identify $F_{\eta_{1}}\left(z_{1}^{\prime} \lambda_{0}+z_{3}^{\prime} \beta_{0}+\alpha_{0}-\cdot\right)$ by

$$
F_{\eta_{1}}\left(z_{1}^{\prime} \lambda_{0}+z_{3}^{\prime} \beta_{0}+\alpha_{0}-\gamma_{0} \cdot\right)=\mathcal{F}^{-1}\left(\frac{\mathcal{F}\left(\partial_{x} P^{11}\left(z_{1}, z_{3}, \cdot\right)\right)}{\mathcal{F}\left(f_{\eta_{2}}(\cdot)\right)}\right)(\cdot) / f_{W}(\cdot) .
$$

Similarly, we can identify

$$
F_{\eta_{1}}\left(z_{1}^{\prime} \lambda_{0}+z_{3}^{\prime} \beta_{0}-\gamma_{0} \cdot\right)=\mathcal{F}^{-1}\left(\frac{\mathcal{F}\left(-\partial_{x} P^{10}\left(z_{1}, z_{3}, \cdot\right)\right)}{\mathcal{F}\left(f_{\eta_{2}}(\cdot)\right)}\right)(\cdot) / f_{W}(\cdot) .
$$


Because $F_{\eta_{1}}(\cdot)$ is monotone increasing, we have

$$
\begin{aligned}
& \mathcal{F}^{-1}\left(\frac{\mathcal{F}\left(\partial_{x} P^{11}\left(z_{1}, z_{3}, \cdot\right)\right)}{\mathcal{F}\left(f_{\eta_{2}}(\cdot)\right)}\right)(w) / f_{W}(w)=\mathcal{F}^{-1}\left(\frac{\mathcal{F}\left(-\partial_{x} P^{10}\left(\tilde{z}_{1}, \tilde{z}_{3}, \cdot\right)\right)}{\mathcal{F}\left(f_{\eta_{2}}(\cdot)\right)}\right)(\tilde{w}) / f_{W}(\tilde{w}) \\
\Longleftrightarrow & \alpha_{0}+\left(z_{1}-\tilde{z}_{1}\right)^{\prime} \lambda_{0}+\left(z_{3}-\tilde{z}_{3}\right)^{\prime} \beta_{0}-\gamma_{0}(w-\tilde{w})=0
\end{aligned}
$$

Then, by Assumption B7, we can find $\left(z_{1}^{(l)}, z_{3}^{(l)}, w^{(l)}\right)_{l=1}^{d}$ and $\left(\tilde{z}_{1}^{(l)}, \tilde{z}_{3}^{(l)}, \tilde{w}^{(l)}\right)_{l=1}^{d}$ such that

$$
\operatorname{rank}\left(\begin{array}{ccc}
1 & \cdots & 1 \\
z_{1,-1}^{(1)}-\tilde{z}_{1,-1}^{(1)} & \cdots & z_{1,-1}^{(d)}-\tilde{z}_{1,-1}^{(d)} \\
z_{3}^{(1)}-\tilde{z}_{3}^{(1)} & \cdots & z_{3}^{(d)}-\tilde{z}_{3}^{(d)} \\
w^{(1)}-\tilde{w}^{(1)} & \cdots & w^{(d)}-\tilde{w}^{(d)}
\end{array}\right)=d .
$$

Then, we can identify $\left(\alpha_{0}, \lambda_{0}, \beta_{0}, \gamma_{0}\right)$ by solving the linear system that

$$
\begin{gathered}
\alpha_{0}+\left(z_{1,-1}^{(1)}-\tilde{z}_{1,-1}^{(1)}\right)^{\prime} \lambda_{0,-1}+\left(z_{3}^{(1)}-\tilde{z}_{3}^{(1)}\right)^{\prime} \beta_{0}-\gamma_{0}\left(w^{(1)}-\tilde{w}^{(1)}\right)=\tilde{z}_{1,1}^{(1)}-z_{1,1}^{(1)}, \\
\vdots \\
\alpha_{0}+\left(z_{1,-1}^{(d)}-\tilde{z}_{1,-1}^{(d)}\right)^{\prime} \lambda_{0,-1}+\left(z_{3}^{(d)}-\tilde{z}_{3}^{(d)}\right)^{\prime} \beta_{0}-\gamma_{0}\left(w^{(d)}-\tilde{w}^{(d)}\right)=\tilde{z}_{1,1}^{(d)}-z_{1,1}^{(d)} .
\end{gathered}
$$

This concludes the proof.

\section{J Proof of Theorem F.1}

Recall we defined our two step rank estimator as follows: Letting $\hat{\theta}$ denote $(\hat{\alpha}, \hat{\gamma})$, our estimator is of the form:

$$
\hat{\theta}=\arg \max _{\theta} \hat{Q}_{n}(\theta) \equiv \sum_{i \neq j} \hat{g}_{i, j}(\theta)
$$

in which

$$
\begin{aligned}
\hat{g}_{i, j}(\theta) & =\left[\mathbf{1}\left\{\partial_{2} \hat{P}^{11}\left(X_{1, i}, X_{i}\right) / \hat{f}_{V}\left(X_{i}\right)+\partial_{2} \hat{P}^{10}\left(X_{1, j}, X_{j}\right) / \hat{f}_{V}\left(X_{j}\right) \geq 0\right\} \mathbf{1}\left\{\Phi\left(X_{1, i}, X_{i}, X_{1, j}, X_{j} ; \theta\right) \geq 0\right\}\right. \\
& \left.+\mathbf{1}\left\{\partial_{2} \hat{P}^{11}\left(X_{1, i}, X_{i}\right) / \hat{f}_{V}\left(X_{i}\right)+\partial_{2} \hat{P}^{10}\left(X_{1, j}, X_{j}\right) / \hat{f}_{V}\left(X_{j}\right)<0\right\} \mathbf{1}\left\{\Phi\left(X_{1, i}, X_{i}, X_{1, j}, X_{j} ; \theta\right)<0\right\}\right],
\end{aligned}
$$

with

$$
\Phi\left(x_{1}, x, \tilde{x}_{1}, \tilde{x} ; \theta\right)=x_{1}+\alpha-\gamma x-\left(\tilde{x}_{1}-\gamma \tilde{x}\right)
$$


We first show consistency of the rank estimator. To do so we first define the objective function $Q_{n, 2}^{i f}(\theta)$, defined as

$$
Q_{n, 2}^{i f}(\theta) \equiv \sum_{i \neq j} g_{i, j}(\theta)
$$

where

$$
\begin{aligned}
g_{i, j}(\theta) & =\left[\mathbf{1}\left\{\partial_{2} P^{11}\left(X_{1, i}, X_{i}\right) / f_{V}\left(X_{i}\right)+\partial_{2} P^{10}\left(X_{1, j}, X_{j}\right) / f_{V}\left(X_{j}\right) \geq 0\right\} \mathbf{1}\left\{\Phi\left(X_{1, i}, X_{i}, X_{1, j}, X_{j} ; \theta\right) \geq 0\right\}\right. \\
& \left.+\mathbf{1}\left\{\partial_{2} P^{11}\left(X_{1, i}, X_{i}\right) / f_{V}\left(X_{i}\right)+\partial_{2} P^{10}\left(X_{1, j}, X_{j}\right) / f_{V}\left(X_{j}\right)<0\right\} \mathbf{1}\left\{\Phi\left(X_{1, i}, X_{i}, X_{1, j}, X_{j} ; \theta\right)<0\right\}\right],
\end{aligned}
$$

Since $g_{i, j}$ is bounded by $1 \forall i, j$, and our random sampling assumption, we have for each $\theta$,

$$
Q_{n, 2}^{i f}(\theta) \stackrel{p}{\rightarrow} E\left[g_{i, j}(\theta)\right] \equiv \Gamma_{0}(\theta)
$$

Furthermore, by Assumptions RK2, RK3 we can extend this result to converging uniformly over $\theta \in \Theta$ (see, e.g. Sherman (1994a), Sherman (1993).) $\Gamma_{0}(\theta)$ is continuous in $\theta$ by Assumptions RK2,RK3, and uniquely maximized at $\theta=\theta_{0}$ by our identification result in Theorem 2.1. Along with Assumption RK1, the infeasible estimator, defined as the maximizer of $Q_{n, 2}^{i f}(\theta)$ converges in probability to $\theta_{0}$ by, for example Theorem 2.1 in Newey and McFadden (1994). To show consistency of the feasible estimator, where we first estimate the choice probability functions and their derivatives nonparametrically, we only now need to show the two objective functions converged to each other uniformly in $\theta \in \Theta$. Consistency of the first stage estimators follows from Assumptions RK3-RK5, see for example Henderson, Li, Parmeter, and Yao (2015). However, this does not immediately imply convergence of the difference in feasible and infeasible objective functions since the nonparametric estimators are inside indicator functions so the continuous mapping theorem does immediately not apply. Nonetheless the desired result can still be attained in one of two ways. One would be to replace indicator functions with smooth distribution functions in a fashion analogous to Horowitz (1992). This would have the disadvantage of introducing tuning parameters, but another approach would be to replace the indicator functions with their conditional expectations, and note that the conditional expectations are smooth functions using Assumption RK2, RK3. To see why, let $\hat{m}\left(x_{i}\right)$ be a nonparametric estimator of a function $m\left(x_{i}\right)$, which is assumed to be smooth. We evaluate the plim of

$$
I\left[\hat{m}\left(x_{i}\right)>0\right]-I\left[m\left(x_{i}\right)>0\right]=I\left[\hat{m}\left(x_{i}\right)>0, m\left(x_{i}\right)<0\right]-I\left[\hat{m}\left(x_{i}\right)<0, m\left(x_{i}\right)>0\right]
$$

we show that the first term converges in probability to 0 as identical arguments can be used for the second term. Let $\varepsilon>0$ be given; $P\left(I\left[\hat{m}\left(x_{i}\right)>0, m\left(x_{i}\right)<0\right]>\varepsilon\right) \leq E\left[I\left[\hat{m}\left(x_{i}\right)>0, m\left(x_{i}\right)<0\right] / \varepsilon\right.$ 
by Markov's inequality. But the expectation in the numerator on the right hand side is

$$
P\left(\hat{m}\left(x_{i}\right)>0, m\left(x_{i}\right)<0\right)=P\left(\hat{m}\left(x_{i}\right)>0, m\left(x_{i}\right) \leq-\delta_{n}\right)+P\left(\hat{m}\left(x_{i}\right)>0, m\left(x_{i}\right) \in\left(-\delta_{n}, 0\right)\right)
$$

where $\delta_{n}$ is a sequence of positive numbers converging to 0 , at a slow rate, e.g.( $\left.\log n^{-1}\right)$. The first term on the right hand side is bounded above by

$$
P\left(\left|\hat{m}\left(x_{i}\right)-m\left(x_{i}\right)\right|>\delta_{n}\right) \leq P\left(\|\hat{m}(\cdot)-m(\cdot)\|>\delta_{n}\right)
$$

where the notation $\|\hat{m}(\cdot)-m(\cdot)\|$ above denotes the sup norm over $x_{i}$. The right hand side probability above will be sufficiently small for $n$ large enough by the rate of convergence of the nonparametric estimator. The second term, $P\left(\hat{m}\left(x_{i}\right)>0, m\left(x_{i}\right) \in\left(-\delta_{n}, 0\right)\right)$, is bounded above by $P\left(m\left(x_{i}\right) \in\left(-\delta_{n}, 0\right)\right)$ which by the smoothness of $m\left(x_{i}\right)$ converges to 0 , and hence can be made arbitrarily small.

To derive the rate of convergence and limiting distribution theory for the feasible estimator where we first estimate choice probability functions and their derivatives nonparametrically, we expand the nonparametric estimators around true functions that are inside the indicator function in $Q_{n 2}$. Then we can follow the approach in Sherman (1994b). Having already established consistency of the estimator, we will first establish root- $n$ consistency and then asymptotic normality. For root- $n$ consistency we will apply Theorem 1 of Sherman (1994b) and so here we change notation to deliberately stay as close as possible to his. We will actually apply this theorem twice, first establishing a slower than root- $n$ consistency result and then root- $n$ consistency. Keeping our notation deliberately as close as possible to Sherman(1994b), here replacing our second stage rank objective function $\hat{Q}_{2, n}(\theta)$ with $\hat{\mathcal{G}}_{n}(\theta)$, our infeasible objective function $Q_{n, 2}^{i f}(\theta)$ with $\mathcal{G}_{n}(\theta)$, and denoting our limiting objective function, previously denoted by $\Gamma_{0}(\theta)$, by $\mathcal{G}(\theta)$. We have the following theorem:

Theorem J.1. (From Theorem 1 in Sherman (1994b)).

If $\delta_{n}$ and $\varepsilon_{n}$ are sequences of positive numbers converging to 0 , and

1. $\hat{\theta}-\theta_{0}=o_{p}\left(\delta_{n}\right)$

2. There exists a neighborhood of $\theta_{0}$ and a constant $\kappa>0$ such that $\mathcal{G}(\theta)-\mathcal{G}\left(\theta_{0}\right) \geq \kappa\left\|\theta-\theta_{0}\right\|^{2}$ for all $\theta$ in this neighborhood.

3. Uniformly over $O_{p}\left(\delta_{n}\right)$ neighborhoods of $\theta_{0}$

$$
\hat{\mathcal{G}}_{n}(\theta)=\mathcal{G}(\theta)+O_{p}\left(\left\|\theta-\theta_{0}\right\| / \sqrt{n}\right)+o_{p}\left(\left\|\theta-\theta_{0}\right\|^{2}\right)+O_{p}\left(\varepsilon_{n}\right)
$$

then $\hat{\theta}-\theta_{0}=O_{p}\left(\max \left(\varepsilon^{1 / 2}, n^{-1 / 2}\right)\right)$. 
Once we use this theorem to establish the rate of convergence of our rank estimator, we can attain limiting distribution theory, which will follow from the following theorem:

Theorem J.2. (From Theorem 2 in Sherman (1994b)). Suppose $\hat{\theta}$ is $\sqrt{n}$-consistent for $\theta_{0}$, an interior point of $\Theta$. Suppose also that uniformly over $O_{p}\left(n^{-1 / 2}\right)$ neighborhoods of $\theta_{0}$,

$$
\hat{\mathcal{G}}_{n}(\theta)=\frac{1}{2}\left(\theta-\theta_{0}\right)^{\prime} V\left(\theta-\theta_{0}\right)+\frac{1}{\sqrt{n}}\left(\theta-\theta_{0}\right)^{\prime} W_{n}+o_{p}(1 / n)
$$

where $V$ is a negative definite matrix, and $W_{n}$ converges in distribution to a $N(0, \Delta)$ random vector. Then

$$
\sqrt{n}\left(\hat{\theta}-\theta_{0}\right) \Rightarrow N\left(0, V^{-1} \Delta V^{-1}\right)
$$

We first turn attention to applying Theorem J.1 to derive the rate of convergence of our estimator. Having already established consistency of our rank estimator, we turn attention to the second condition in Theorem J.1. To show the second condition, we will first derive an expansion for $\mathcal{G}(\theta)$ around $\mathcal{G}\left(\theta_{0}\right)$. We denote that even though $\mathcal{G}_{n}(\theta)$ is not differentiable in $\theta, \mathcal{G}(\theta)$ is sufficiently smooth for Taylor expansions to apply as the expectation operator is a smoothing operator and the smoothness conditions in Assumptions RK2, RK3. Taking a second order expansion of $\mathcal{G}(\theta)$ around $\mathcal{G}\left(\theta_{0}\right)$, we obtain

$$
\mathcal{G}(\theta)=\mathcal{G}\left(\theta_{0}\right)+\nabla_{\beta} \mathcal{G}\left(\theta_{0}\right)^{\prime}\left(\theta-\theta_{0}\right)+\frac{1}{2}\left(\theta-\theta_{0}\right)^{\prime} \nabla_{\theta \theta} \mathcal{G}\left(\theta^{*}\right)\left(\theta-\theta_{0}\right)
$$

where $\nabla_{\theta}$ and $\nabla_{\theta \theta}$ denote first and second derivative operators and $\theta^{*}$ denotes an intermediate value. We note that the first two terms of the right hand side of the above equation are 0 , the first by how we defined the objective function, and the second by our identification result in Theorem 2.1. Define

$$
V \equiv \nabla_{\theta \theta} \mathcal{G}\left(\theta_{0}\right)
$$

and $V$ is positive definite by Assumption A3, so we have

$$
\left(\theta-\theta_{0}\right)^{\prime} \nabla_{\theta \theta} \mathcal{G}\left(\theta_{0}\right)\left(\theta-\theta_{0}\right)>0
$$

$\nabla_{\theta \theta} \mathcal{G}(\theta)$ is also continuous at $\theta=\theta_{0}$ by Assumptions RK2 and RK3, so there exists a neighborhood of $\theta_{0}$ such that for all $\theta$ in this neighborhood, we have

$$
\left(\theta-\theta_{0}\right)^{\prime} \nabla_{\theta \theta} \mathcal{G}(\theta)\left(\theta-\theta_{0}\right)>0
$$

which suffices for the second condition to hold.

To show the third condition in Theorem J.1, we next establish the form of the remainder term when we replace nonparametric estimators with the true functions they are estimating. Specifically 
we wish to evaluate the difference between

$$
\begin{aligned}
& {\left[\mathbf{1}\left\{\partial_{2} \hat{P}^{11}\left(X_{1, i}, X_{i}\right) / \hat{f}_{V}\left(X_{i}\right)+\partial_{2} \hat{P}^{10}\left(X_{1, j}, X_{j}\right) / \hat{f}_{V}\left(X_{j}\right) \geq 0\right\} \mathbf{1}\left\{\Phi\left(X_{1, i}, X_{i}, X_{1, j}, X_{j} ; \theta\right) \geq 0\right\}\right.} \\
+\quad & \mathbf{1}\left\{\partial_{2} \hat{P}^{11}\left(X_{1, i}, X_{i}\right) / \hat{f}_{V}\left(X_{i}\right)+\partial_{2} \hat{P}^{10}\left(X_{1, j}, X_{j}\right) / \hat{f}_{V}\left(X_{j}\right)<0\right\} \mathbf{1}\left\{\Phi\left(X_{1, i}, X_{i}, X_{1, j}, X_{j} ; \theta\right)<0\right\}
\end{aligned}
$$

and

$$
\begin{aligned}
& {\left[\mathbf{1}\left\{\partial_{2} P^{11}\left(X_{1, i}, X_{i}\right) / f_{V}\left(X_{i}\right)+\partial_{2} P^{10}\left(X_{1, j}, X_{j}\right) / f_{V}\left(X_{j}\right) \geq 0\right\} \mathbf{1}\left\{\Phi\left(X_{1, i}, X_{i}, X_{1, j}, X_{j} ; \theta\right) \geq 0\right\}\right.} \\
+\quad & \mathbf{1}\left\{\partial_{2} P^{11}\left(X_{1, i}, X_{i}\right) / f_{V}\left(X_{i}\right)+\partial_{2} P^{10}\left(X_{1, j}, X_{j}\right) / f_{V}\left(X_{j}\right)<0\right\} \mathbf{1}\left\{\Phi\left(X_{1, i}, X_{i}, X_{1, j}, X_{j} ; \theta\right)<0\right\}
\end{aligned}
$$

To establish a representation for this difference, we first simplify notation we write the expressions as:

$$
\begin{array}{r}
I\left[\hat{m}_{1}\left(\mathbf{x}_{i}\right)+\hat{m}_{2}\left(\mathbf{x}_{j}\right) \geq 0\right] I\left[\Delta \mathbf{x}_{i j}^{\prime} \theta \geq 0\right] \\
+\quad I\left[\hat{m}_{1}\left(\mathbf{x}_{i}\right)+\hat{m}_{2}\left(\mathbf{x}_{j}\right)<0\right] I\left[\Delta \mathbf{x}_{i j}^{\prime} \theta<0\right]
\end{array}
$$

and

$$
\begin{array}{r}
I\left[m_{1}\left(\mathbf{x}_{i}\right)+m_{2}\left(\mathbf{x}_{j}\right) \geq 0\right] I\left[\Delta \mathbf{x}_{i j}^{\prime} \theta \geq 0\right] \\
+\quad I\left[m_{1}\left(\mathbf{x}_{i}\right)+m_{2}\left(\mathbf{x}_{j}\right)<0\right] I\left[\Delta \mathbf{x}_{i j}^{\prime} \theta<0\right]
\end{array}
$$

respectively, where here $\mathbf{x}_{i}$ denotes the separate components of $x_{1 i}, x_{i}$, and analogous for $\mathbf{x}_{j}$. We first explore

$$
\left(I\left[\hat{m}_{1}\left(\mathbf{x}_{i}\right)+\hat{m}_{2}\left(\mathbf{x}_{j}\right) \geq 0\right]-I\left[m_{1}\left(\mathbf{x}_{i}\right)+m_{2}\left(\mathbf{x}_{j}\right) \geq 0\right]\right) I\left[\Delta \mathbf{x}_{i j}^{\prime} \theta \geq 0\right]
$$

for each $i, j$ inside the double summation:

$$
\frac{1}{n(n-1)} \sum_{i \neq j}\left(I\left[\hat{m}_{1}\left(\mathbf{x}_{i}\right)+\hat{m}_{2}\left(\mathbf{x}_{j}\right) \geq 0\right]-I\left[m_{1}\left(\mathbf{x}_{i}\right)+m_{2}\left(\mathbf{x}_{j}\right) \geq 0\right]\right) I\left[\Delta \mathbf{x}_{i j}^{\prime} \theta \geq 0\right]
$$

An immediate technical difficulty that arises with the above term is the presence of a nonparametric estimator inside the indicator function above. A simple approach to deal with this would be to replace the indicator function with a smoothed indicator function in a fashion analogous to Horowitz (1992), under appropriate conditions on the kernel function and smoothing parameter. Such an approach is not necessary as long as the nonparametric estimator $\hat{m}_{1}\left(x_{i}\right)$ is asymptotically normal, and asymptotically centered at $m_{1}\left(x_{i}\right)$, which will be the case with our proposed kernel estimator of the probability function and its derivative. In either approach (smoothed indicator or 
not) we can show that (J.15) can be represented as:

$$
\frac{1}{n(n-1)} \sum_{i \neq j} \phi(0) f_{m_{i j}}(0)\left(\left(\hat{m}_{1}\left(\mathbf{x}_{i}\right)-m_{1}\left(\mathbf{x}_{i}\right)\right)+\left(\hat{m}_{2}\left(\mathbf{x}_{j}\right)-m_{2}\left(\mathbf{x}_{j}\right)\right)\right) I\left[\Delta \mathbf{x}_{i j}^{\prime} \theta \geq 0\right]+o_{p}\left(n^{-1}\right)
$$

where $\phi(0)$ denotes the standard normal pdf evaluated at $0, f_{m_{i j}}(0)$ denotes the density function of $m_{1}\left(\mathbf{x}_{i}\right)+m_{2}\left(\mathbf{x}_{j}\right)$ evaluated at 0 , and the $o_{p}\left(n^{-1}\right)$ term is uniform in $\theta$ lying in $o_{p}(1)$ neighborhoods of $\theta_{0}$. Therefore, uniformly for $\theta$ in an $o_{p}(1)$ neighborhood of $\theta_{0}$, this remainder term converges to 0 at the rate of convergence of the first stage nonparametric estimator, which under Assumptions RK3, RK4, RK5, is $o_{p}\left(n^{-1 / 4}\right)$. Thus by repeated application of Theorem J.1, we can conclude that the estimator is root- $n$ consistent. To show that the estimator is also asymptotically normal, we will first derive a linear representation for the term:

$$
\frac{1}{n(n-1)} \sum_{i \neq j} \phi(0) f_{m_{i j}}(0)\left(\hat{m}_{1}\left(\mathbf{x}_{i}\right)-m_{1}\left(\mathbf{x}_{i}\right)\right) I\left[\Delta \mathbf{x}_{i j}^{\prime} \theta \geq 0\right]
$$

As this term is linear in the nonparametric estimator $\hat{m}_{1}\left(x_{i}\right)$, the desired linear representation follows from arguments used in Khan (2001). One slight difference here compared to Khan (2001) is that here our nonparametric estimators and estimands are each ratios of derivatives. Nonetheless, after linearizing these ratios as done in, e.g. Newey and McFadden (1994). Specifically, we have that J.17 can be expressed as:

$$
\begin{aligned}
& \frac{1}{n(n-1)} \sum_{i \neq j} \phi(0) f_{m_{i j}}(0) \frac{1}{m_{1 d e n}\left(\mathbf{x}_{i}\right)}\left(\hat{m}_{1 n u m}\left(\mathbf{x}_{i}\right)-m_{1 n u m}\left(\mathbf{x}_{i}\right)\right) I\left[\Delta \mathbf{x}_{i j}^{\prime} \theta \geq 0\right] \\
& -\frac{1}{n(n-1)} \sum_{i \neq j} \phi(0) f_{m_{i j}}(0) \frac{m_{1 n u m}\left(\mathbf{x}_{i}\right)}{m_{1 \operatorname{den}}\left(\mathbf{x}_{i}\right)^{2}}\left(\hat{m}_{1 \operatorname{den}}\left(\mathbf{x}_{i}\right)-m_{1 \operatorname{den}}\left(\mathbf{x}_{i}\right)\right) I\left[\Delta \mathbf{x}_{i j}^{\prime} \theta \geq 0\right]
\end{aligned}
$$

where $\hat{m}_{1 \text { num }}\left(\mathbf{x}_{i}\right)$ denotes the numerator $\left\{\partial_{2} \hat{P}^{11}\left(X_{1, i}, X_{i}\right)\right\}$, the estimator of $m_{1 \text { num }}\left(\mathbf{x}_{i}\right)$ which denotes $\left\{\partial_{2} P^{11}\left(X_{1, i}, X_{i}\right)\right\}$, and $\hat{m}_{1 \text { den }}\left(\mathbf{x}_{i}\right)$ denotes the denominator $\hat{f}_{V}\left(X_{i}\right)$, the estimator of $m_{1 d e n}\left(\mathbf{x}_{i}\right)$ which denotes $f_{V}\left(X_{i}\right)$.

Plugging in the definitions of the kernel estimators of $\hat{m}_{1 \text { num }}\left(\mathbf{x}_{i}\right)$, and $\hat{m}_{1 \text { den }}\left(\mathbf{x}_{i}\right)$, results in a third order process. Using arguments in Khan (2001) and Powell, Stock, and Stoker (1989) we can express the third order $U$ process as a second order $U$ process plus an asymptotically negligible remainder term. This is of the form:

$$
\frac{1}{n} \sum_{i=1}^{n} \phi(0) \frac{\ell\left(x_{i}\right)}{m_{1 \operatorname{den}}\left(\mathbf{x}_{i}\right)}\left(y_{1 i}-m_{1 \text { num }}\left(\mathbf{x}_{i}\right)\right) E\left[I\left[f_{m_{i j}}(0) \Delta \mathbf{x}_{i j}^{\prime} \theta \geq 0\right] \mid x_{i}\right]
$$

where $\ell\left(x_{i}\right) \equiv \frac{-f_{X}^{\prime}\left(x_{i}\right)}{f_{X}\left(x_{i}\right)}$. We note that the function $E\left[f_{m_{i j}}(0) I\left[\Delta \mathbf{x}_{i j}^{\prime} \theta \geq 0\right] \mid x_{i}\right]$, which we denote here by $\mathcal{H}\left(x_{i}, \theta\right)$ is a smooth function in $\theta$. We will use this feature to expand $\mathcal{H}\left(x_{i}, \theta\right)$ around 
$\mathcal{H}\left(x_{i}, \theta_{0}\right)$. Analogous arguments can be used to attain a linear representation of (J.19), which is of the form:

$$
\frac{1}{n} \sum_{i=1}^{n} \phi(0) \frac{\ell_{2}\left(x_{1 i}\right) m_{1 n u m}\left(\mathbf{x}_{i}\right)}{m_{1 \operatorname{den}}\left(\mathbf{x}_{i}\right)^{2}}\left(y_{2 i}-m_{1 \operatorname{den}}\left(\mathbf{x}_{i}\right)\right) E\left[I\left[f_{m_{i j}}(0) \Delta \mathbf{x}_{i j}^{\prime} \theta \geq 0\right] \mid x_{i}\right]
$$

where $\ell_{2}\left(x_{1 i}\right) \equiv \frac{-f_{X_{1}}^{\prime}\left(x_{1 i}\right)}{f_{X}\left(x_{1 i}\right)}$. Grouping (J.20) and (J.21) we have

$$
\frac{1}{n} \sum_{i=1}^{n} \phi(0) \frac{1}{m_{1 \text { den }}\left(\mathbf{x}_{i}\right)}\left\{\ell\left(x_{i}\right)\left(y_{1 i}-m_{1 n u m}\left(\mathbf{x}_{i}\right)\right)-\frac{m_{1 \text { num }}\left(\mathbf{x}_{i}\right)}{m_{1 \text { den }}\left(\mathbf{x}_{i}\right)} \ell_{2}\left(x_{1 i}\right)\left(y_{2 i}-m_{1 \text { den }}\left(\mathbf{x}_{i}\right)\right)\right\} \mathcal{H}\left(x_{i}, \theta\right)
$$

Note that by Assumptions RK2, RK3, $\mathcal{H}\left(x_{i}, \theta\right)$ is smooth in $\theta$ implying the expansion

$$
\mathcal{H}\left(x_{i}, \theta\right)=\mathcal{H}\left(x_{i}, \theta_{0}\right)+\nabla_{\theta} \mathcal{H}\left(x_{i}, \theta_{0}\right)^{\prime}\left(\theta-\theta_{0}\right)
$$

Thus we can express (J.22) as the which we note is a mean 0 sum

$$
\frac{1}{n} \sum_{i=1}^{n} \psi_{1 r n k i}\left(\theta-\theta_{0}\right)
$$

where

$$
\psi_{1 r n k i}=\phi(0) \frac{1}{m_{1 \operatorname{den}}\left(\mathbf{x}_{i}\right)}\left\{\ell\left(x_{i}\right)\left(y_{1 i}-m_{1 \text { num }}\left(\mathbf{x}_{i}\right)\right)-\frac{m_{1 \text { num }}\left(\mathbf{x}_{i}\right)}{m_{1 \operatorname{den}}\left(\mathbf{x}_{i}\right)} \ell_{2}\left(x_{1 i}\right)\left(y_{2 i}-m_{1 \operatorname{den}}\left(\mathbf{x}_{i}\right)\right)\right\} \nabla_{\theta} \mathcal{H}\left(x_{i}, \theta_{0}\right)
$$

We can use identical arguments to attain a linear representation for the $U$ - process:

$$
\frac{1}{n(n-1)} \sum_{i \neq j} \phi(0) f_{m_{i j}}(0)\left(\hat{m}_{2}\left(\mathbf{x}_{j}\right)-m_{2}\left(\mathbf{x}_{j}\right)\right) I\left[\Delta \mathbf{x}_{i j}^{\prime} \theta \geq 0\right]
$$

where $\hat{m}_{2}\left(\mathbf{x}_{j}\right)$ is also a ratio of nonparametric estimators where here the numerator is $\hat{m}_{2 n}\left(\mathbf{x}_{j}\right)$ denoting $\left\{\partial_{2} \hat{P}^{10}\left(X_{1, j}, X_{j}\right)\right\}$, the estimator of $m_{2 n}\left(\mathbf{x}_{2}\right)$ which denotes $\left\{\partial_{2} P^{10}\left(X_{1, j}, X_{j}\right)\right\}$, and $\hat{m}_{2 d}\left(\mathbf{x}_{j}\right)$ denotes the denominator $\hat{f}_{V}\left(X_{j}\right)$, the estimator of $m_{1 d e n}\left(\mathbf{x}_{j}\right)$ which denotes $f_{V}\left(X_{j}\right)$.

and by using identical arguments it too can be represented as a mean 0 sum denoted here by

$$
\frac{1}{n} \sum_{i=1}^{n} \psi_{2 r n k i}
$$

where $\psi_{2 r n k i}$ is defined as:

Finally after grouping the two terms and expanding $\mathcal{H}\left(x_{i}, \theta\right)$ around $\mathcal{H}\left(x_{i}, \theta_{0}\right)$ we get that (J.16) 
can be represented as:

$$
\frac{1}{n} \sum_{i=1}^{n}\left(\psi_{1 r n k i}+\psi_{2 r n k i}\right)^{\prime}\left(\theta-\theta_{0}\right)+o_{p}\left(n^{-1}\right)
$$

Combining our results, from Theorem J.2, we have that

$$
\sqrt{n}\left(\hat{\theta}-\theta_{0}\right) \Rightarrow N\left(0, V^{-1} \Delta V^{-1}\right)
$$

where

$$
V=\nabla_{\theta \theta} \mathcal{G}\left(\theta_{0}\right)
$$

and

$$
\Delta=E\left[\left(\psi_{1 r n k i}+\psi_{2 r n k i}\right)\left(\psi_{1 r n k i}+\psi_{2 r n k i}\right)^{\prime}\right]
$$

\section{K Model with Two Idiosyncratic Shocks}

In this section, we focus on the identification of $\left(\alpha_{0}, \gamma_{0}\right)$ in the "condensed" model that $X_{1}=$ $Z_{1}^{\prime} \lambda_{0}+Z_{3}^{\prime} \beta_{0}$ is observed and

$$
\begin{aligned}
& Y_{1}=\mathbf{1}\left\{X_{1}+\alpha_{0} Y_{2}-U \geq 0\right\} \\
& Y_{2}=\mathbf{1}\{X-V \geq 0\}
\end{aligned}
$$

with the understanding that $\left(\lambda_{0}, \beta_{0}\right)$ can be identified jointly with $\alpha_{0}$ and $\gamma_{0}$, as shown in Theorems 2.1 and 3.1. We further impose $U=\gamma_{0} W+\eta_{1}, V=W+\eta_{2}$, and $\left(W, \eta_{1}, \eta_{2}\right)$ are mutually independent. First we consider the case $\gamma_{0}=1$ and $X_{1}$ is binary, because even in this context, for the baseline case with one idiosyncratic shock, we can identify $\alpha_{0}$. But identification of $\alpha_{0}$ becomes more difficult in this model without the help of repeated measurements, as established in the following theorem.

Theorem K.1. Suppose (K.31) holds, $\gamma_{0}$ is known to be one, $X_{1}$ is binary, and $W$ has a bounded support $[-b,-a]$ such that $0.5>b-a$ and $1-(b-a)>\alpha_{0}>b-a$, then $\alpha_{0}$ is not point identified.

This nonidentification result motivates imposing additional structure on $W$, and we consider the following model

C1 $U=\gamma_{0} W+\eta_{1}$ and $V=\sigma_{0} W+\eta_{2}$.

C2 $W$ is standard normally distributed. 
C3 $W, \eta_{1}$ and $\eta_{2}$ are mutually independent.

C4 $X$ has full support.

C5 Denote the density of $\eta_{2}$ as $f_{\eta_{2}}$, then $f_{\eta_{2}}$ does not have a Gaussian component in the sense that

$$
f_{\eta_{2}} \in \mathcal{G}=\left\{g \text { is a density on } \Re \text { s.t. }: g=g^{\prime} * \phi_{\sigma} \text { for some density } g^{\prime} \text { implies that } \sigma=0\right\} \text {, }
$$

where $\phi_{\sigma}$ is the density for a normal distribution with zero mean and $\sigma^{2}$ variance.

Assumption C5 effectively assumes that the distribution of $\eta_{2}$ has tail properties different from those of a normal distribution. This type of assumption is made in the deconvolution literature as it is necessary for identification of the target density when the error distribution is not completely known- see, e.g., Butucea and Matias (2005). ${ }^{3}$ The importance of non-normality in factor models goes back to Geary (1942) and Reiersol (1950), who have shown that factor loadings are identified in a linear measurement error model if the factor is not Gaussian. In our case, note $V=\sigma_{0} W+\eta_{2}$ where $W$ is standard normal and the density of $V$ is identified from data. Here we want to identify $\sigma_{0}$ and the density of $\eta_{2}$. If $\eta_{2}$ has a Gaussian component, then

$$
\eta_{2}=\eta_{2}^{\prime}+\tilde{\sigma} \tilde{W}
$$

where $\tilde{W}$ is a standard normal random variable that is independent of $\eta_{2}^{\prime}$ and $W$ and $\tilde{\sigma}>0$. It implies

$$
V=\left(\sigma_{0} W+\tilde{\sigma} \tilde{W}\right)+\eta_{2}^{\prime}
$$

where $\eta_{2}^{\prime}$ does not have a Gaussian component. In addition, note that $\left(\sigma_{0} W+\tilde{\sigma} \tilde{W}\right)=\sqrt{\sigma_{0}^{2}+\tilde{\sigma}^{2}} G$, for some standard normal random variable $G$. Therefore, without Assumption B5, $\sigma_{0}$ is not identified.

Theorem K.2. If Assumptions $\boldsymbol{C 1 - C 5}$ hold, then $\sigma_{0}, \gamma_{0}$ and $\alpha_{0}$ are identified.

Note that this identification result does not require any variation from $X_{1}$, which is in spirit close to the one-factor model in our paper and is different from the identification result in Vytlacil and Yildiz (2007). We also note that this result does not contradict the counterexample in the paper. In the counterexample, we only assume that we know the support of $W$ is bounded. Here we assume that the full density of $W$, and thus, the support of $W$ is known.

\footnotetext{
${ }^{3}$ In fact, based on the results in Butucea and Matias (2005), $W$ can belong to a more general class of known distributions. Furthermore, we note that if $\sigma_{0}$ is known, then Assumption C5 is not necessary.
} 


\section{Proof of Theorem E.1}

Denote $P^{i j}\left(x_{1}, x\right)=\operatorname{Prob}\left(Y_{1}=i, Y_{2}=j \mid X_{1}=x_{1}, X=x\right)$. Then

$$
\begin{aligned}
& P^{11}\left(x_{1}, x\right)=\int_{-\infty}^{x} F_{U}\left(x_{1}+\alpha_{0} \mid V=v\right) f(v) d v \\
& P^{10}\left(\tilde{x}_{1}, x\right)=\int_{x}^{+\infty} F_{U}\left(\tilde{x}_{1} \mid V=v\right) f(v) d v
\end{aligned}
$$

Taking derivatives w.r.t. the second argument of the the LHS function, we have

$$
\begin{aligned}
& \partial_{2} P^{11}\left(x_{1}, x\right)=F_{U}\left(x_{1}+\alpha_{0} \mid V=x\right) f(x) \\
& \partial_{2} P^{10}\left(\tilde{x}_{1}, x\right)=-F_{U}\left(\tilde{x}_{1} \mid V=x\right) f(x) .
\end{aligned}
$$

If $\left|\alpha_{0}\right| \leq b-a$, then there exists a pair $\left(x_{1}, \tilde{x}_{1}\right)$ such that $x_{1}+\alpha_{0}=\tilde{x}_{1}$. This pair can be identified by checking the equation below:

$$
\partial_{2} P^{11}\left(x_{1}, x\right) / f(x)+\partial_{2} P^{10}\left(\tilde{x}_{1}, x\right) / f(x)=0
$$

This concludes the sufficient part.

When $\alpha_{0}<a-b$, for any $\alpha<\alpha_{0}$, we can define

$$
\begin{aligned}
& \tilde{U}=U+\alpha-\alpha_{0} \\
& \text { if } \quad U \leq b+\alpha_{0} \\
& \tilde{U}=U \\
& \text { if } \\
& U>b+\alpha_{0}
\end{aligned}
$$

Then for any $x_{1} \in[a, b]$,

$$
\begin{aligned}
P\left(\tilde{U} \leq x_{1}+\alpha \mid V=v\right) & =P\left(\tilde{U} \leq x_{1}+\alpha, U \leq b+\alpha_{0} \mid V=v\right)+P\left(\tilde{U} \leq x_{1}+\alpha, U>b+\alpha_{0} \mid V=v\right) \\
& =P\left(U \leq x_{1}+\alpha_{0} \mid V=v\right) \\
P\left(\tilde{U} \leq x_{1} \mid V=v\right) & =P\left(\tilde{U} \leq x_{1}, U \leq b+\alpha_{0} \mid V=v\right)+P\left(\tilde{U} \leq x_{1}, U>b+\alpha_{0} \mid V=v\right) \\
& =P\left(U \leq b+\alpha_{0}, U \leq x_{1}+\alpha_{0}-\alpha \mid V=v\right)+P\left(b+\alpha_{0}<U \leq x_{1} \mid V=v\right) \\
& =P\left(U \leq b+\alpha_{0} \mid V=v\right)+P\left(b+\alpha_{0}<U \leq x_{1} \mid V=v\right) \\
& =P\left(U \leq x_{1} \mid V=v\right),
\end{aligned}
$$

where the third equality holds because, since $\alpha_{0}<a-b$ and $\alpha<\alpha_{0}, b+\alpha_{0} \leq x_{1}+\alpha_{0}-\alpha$ for $x_{1} \in[a, b]$. Let $G_{U, V}$ and $G_{\tilde{U}, V}$ be the joint distribution of $(U, V)$ and $(\tilde{U}, V)$ respectively. Then the above calculation with (L.32) imply that $\left(\alpha_{0}, G_{U, V}\right)$ and $\left(\alpha, G_{\tilde{U}, V}\right)$ produce the identical pair $\left(P^{11}\left(x_{1}, x\right), P^{10}\left(x_{1}, x\right)\right)$. In addition, the distribution of $V$ is unchanged so that $P\left(Y_{2}=1 \mid X=x\right)$ is identified from data. Therefore, $\left(\alpha_{0}, G_{U, V}\right)$ and $\left(\alpha, G_{\tilde{U}, V}\right)$ are observationally equivalent. 
Similarly, when $\alpha_{0}>b-a$, for any $\alpha>\alpha_{0}$, we can define

$$
\begin{aligned}
& \tilde{U}=U+\alpha-\alpha_{0} \quad \text { if } \quad U>a+\alpha_{0} \\
& \tilde{U}=U \quad \text { if } \quad U \leq a+\alpha_{0}
\end{aligned}
$$

Then for any $x_{1} \in[a, b]$,

$$
\begin{aligned}
P\left(\tilde{U} \leq x_{1}+\alpha \mid V=v\right) & =P\left(\tilde{U} \leq x_{1}+\alpha, U \leq a+\alpha_{0} \mid V=v\right)+P\left(\tilde{U} \leq x_{1}+\alpha, U>a+\alpha_{0} \mid V=v\right) \\
& =P\left(U \leq a+\alpha_{0} \mid V=v\right)+P\left(a+\alpha_{0}<U \leq x_{1}+\alpha_{0} \mid V=v\right) \\
& =P\left(U \leq x_{1}+\alpha_{0} \mid V=v\right) . \\
P\left(\tilde{U} \leq x_{1} \mid V=v\right) & =P\left(\tilde{U} \leq x_{1}, U \leq a+\alpha_{0} \mid V=v\right)+P\left(\tilde{U} \leq x_{1}, U>a+\alpha_{0} \mid V=v\right) \\
& =P\left(U \leq x_{1} \mid V=v\right),
\end{aligned}
$$

where we use the facts that $x_{1} \leq a+\alpha_{0}$ and $x_{1}-a<\alpha$ for $x_{1} \in[a, b]$. So again, $\left(\alpha_{0}, G_{U, V}\right)$ and $\left(\alpha, G_{\tilde{U}, V}\right)$ are observationally equivalent.

\section{Proof of Theorem E.2}

The sign of $\alpha_{0}$ is identified by the data. In the following, we focus on deriving the results when $\alpha_{0}>b-a$. By the proof of Theorem E.1, we have already shown that all $\alpha>\alpha_{0}$ is in the identified set. Now we consider $\frac{b-a+\alpha_{0}}{2} \leq \alpha<\alpha_{0}$.

$$
\begin{aligned}
& \tilde{U}=U+\alpha-\alpha_{0} \quad \text { if } \quad U>a+\alpha \\
& \tilde{U}=U \quad \text { if } \quad U \leq a+\alpha
\end{aligned}
$$

Then for any $x_{1} \in[a, b]$,

$$
\begin{aligned}
P\left(\tilde{U} \leq x_{1}+\alpha \mid V=v\right) & =P\left(\tilde{U} \leq x_{1}+\alpha, U \leq a+\alpha \mid V=v\right)+P\left(\tilde{U} \leq x_{1}+\alpha, U>a+\alpha \mid V=v\right) \\
& =P(U \leq a+\alpha \mid V=v)+P\left(a+\alpha<U \leq x_{1}+\alpha_{0} \mid V=v\right) \\
& =P\left(U \leq x_{1}+\alpha_{0} \mid V=v\right) . \\
P\left(\tilde{U} \leq x_{1} \mid V=v\right) & =P\left(\tilde{U} \leq x_{1}, U \leq a+\alpha \mid V=v\right)+P\left(\tilde{U} \leq x_{1}, U>a+\alpha \mid V=v\right) \\
& =P\left(U \leq x_{1} \mid V=v\right)+P\left(U \leq x_{1}+\alpha_{0}-\alpha, U>a+\alpha \mid V=v\right) . \\
& =P\left(U \leq x_{1} \mid V=v\right) .
\end{aligned}
$$

Here note that the last equality is because $x_{1}+\alpha_{0}-\alpha \leq b+\alpha_{0}-\alpha \leq a+\alpha$ if $\alpha \geq \frac{b-a+\alpha_{0}}{2}$. Denote $\alpha^{(1)}=\frac{b-a+\alpha_{0}}{2}$. Then we have shown that there exists $U^{(1)}(\alpha)$ which only depends on $\alpha$ such that 
for any $x_{1} \in[a, b]$, any $v$ and any $\alpha_{0}>\alpha \geq \alpha^{(1)}$

$$
\begin{aligned}
& P\left(U^{(1)}(\alpha) \leq x_{1}+\alpha \mid V=v\right)=P\left(U \leq x_{1}+\alpha_{0} \mid V=v\right) \\
& P\left(U^{(1)}(\alpha) \leq x_{1} \mid V=v\right)=P\left(U \leq x_{1} \mid V=v\right)
\end{aligned}
$$

In particular, there exists $U^{(1)}\left(\alpha^{(1)}\right)$ such that

$$
\begin{aligned}
& P\left(U^{(1)}\left(\alpha^{(1)}\right) \leq x_{1}+\alpha^{(1)} \mid V=v\right)=P\left(U \leq x_{1}+\alpha_{0} \mid V=v\right) \\
& P\left(U^{(1)}\left(\alpha^{(1)}\right) \leq x_{1} \mid V=v\right)=P\left(U \leq x_{1} \mid V=v\right) .
\end{aligned}
$$

Now repeating the above construction but replacing $U$ with $U^{(1)}$ and $\alpha_{0}$ with $\alpha^{(1)}$, we have for any $\alpha^{(1)}>\alpha \geq \alpha^{(2)} \equiv \frac{b-a+\alpha^{(1)}}{2}$, there exists $U^{(2)}(\alpha)$ such that for any $x_{1} \in[a, b]$, any $v$ and any $\alpha^{(1)}>\alpha \geq \alpha^{(2)}$

$$
\begin{aligned}
& P\left(U^{(2)}(\alpha) \leq x_{1}+\alpha^{(2)} \mid V=v\right)=P\left(U^{(1)}\left(\alpha^{(1)}\right) \leq x_{1}+\alpha^{(1)} \mid V=v\right)=P\left(U \leq x_{1}+\alpha_{0} \mid V=v\right) \\
& P\left(U^{(2)}(\alpha) \leq x_{1} \mid V=v\right)=P\left(U^{(1)}\left(\alpha^{(1)}\right) \leq x_{1} \mid V=v\right)=P\left(U \leq x_{1} \mid V=v\right) .
\end{aligned}
$$

This concludes that any $\alpha$ such that $\alpha_{0}>\alpha \geq \alpha^{(2)}$ is in the identified set. In general, by repeating the procedure $\mathrm{k}$ times, we have that any $\alpha$ such that

$$
\alpha_{0}>\alpha \geq \alpha^{(k)}=\left(1-\frac{1}{2^{k}}\right)(b-a)+\frac{\alpha_{0}}{2^{k}}
$$

is in the identified set. For any $\alpha>b-a$, there exists some finite $k$ such that $\alpha>\left(1-\frac{1}{2^{k}}\right)(b-a)+\frac{\alpha_{0}}{2^{k}}$. This concludes the result that $\alpha>b-a$ is in the identified set.

Finally, since if $\alpha>b-a, \partial_{2} P^{11}\left(x_{1}, x\right)+\partial_{2} P^{10}\left(\tilde{x}_{1}, x\right)>0$ for all pairs of $\left(x_{1}, x\right)$ and $\left(\tilde{x}_{1}, x\right)$ while, if $\alpha \leq b-a$, at least there exists one pair $\left(x_{1}, x\right)$ and $\left(\tilde{x}_{1}, x\right)$ such that $\partial_{2} P^{11}\left(x_{1}, x\right)+\partial_{2} P^{10}\left(\tilde{x}_{1}, x\right) \leq 0$. This implies $\alpha \leq b-a$ is not in the identified set. Therefore, the sharp identified set when $\alpha_{0}>b-a$ is $(b-a, \infty)$.

When $\alpha_{0}<a-b$, a symmetric argument implies that the identified set is $(-\infty, a-b)$.

\section{N Proof of Theorem K.1}

Our first result for this model illustrates how identification can become more difficult. In our first result for this model, we show when $-W$ has a bounded support, say $[a, b]$, then $\alpha_{0}$ is not identified if $\alpha_{0}>b-a$. To establish this, consider an impostor $\alpha$ such that $\alpha<\alpha_{0}$. In addition, we consider the case where $\alpha_{0}-\alpha+b<\alpha_{0}+a$ and $\alpha+b<a+1$. Such $\alpha$ exists because of the fact that $1-(b-a)>\alpha_{0}>b-a$. Let $\Delta=\alpha_{0}-\alpha$ and $\left(\tilde{W}, \tilde{\eta}_{1}, \tilde{\eta}_{2}\right)$ be mutually independent such that $\tilde{W}$ is 
distributed as $W-\Delta, \tilde{\eta}_{2}$ is distributed as $\eta_{2}-\Delta$, and

$$
F_{\tilde{\eta}_{1}}(e)=\left\{\begin{array}{l}
F_{\eta_{1}}(e) \\
F_{\eta_{1}}(a) \\
F_{\eta_{1}}(e-\Delta) \\
\frac{\alpha_{0}+a-e}{\alpha_{0}+a-b-\Delta} F_{\eta_{1}}(b)+\frac{e-b-\Delta}{\alpha_{0}+a-b-\Delta} F_{\eta_{1}}\left(\alpha_{0}+a\right) \\
F_{\eta_{1}}(e) \\
F_{\eta_{1}}\left(\alpha_{0}+b\right)+\frac{e-\alpha_{0}-b}{a+1+\Delta-\alpha_{0}-b}\left(F_{\eta_{1}}(a+1)-F_{\eta_{1}}\left(\alpha_{0}+b\right)\right) \\
F_{\eta_{1}}(e-\Delta) \\
F_{\eta_{1}}(b+1)+\frac{e-(b+\Delta+1)}{a+\alpha_{0}-b-\Delta}\left(F_{\eta_{1}}\left(a+\alpha_{0}+1\right)-F_{\eta_{1}}(b+1)\right) \\
F_{\eta_{1}}(e)
\end{array}\right.
$$

on $e \leq a$, on $\eta_{1} \in(a, a+\Delta]$, on $e \in(a+\Delta, b+\Delta]$, on $e \in\left(b+\Delta, \alpha_{0}+a\right]$, on $e \in\left(\alpha_{0}+a, \alpha_{0}+b\right)$, on $e \in\left(\alpha_{0}+b, a+1+\Delta\right]$, on $e \in(a+\Delta+1, b+\Delta+1]$, on $e \in\left(b+\Delta+1, a+\alpha_{0}+1\right]$, on $e>a+\alpha_{0}+1$.

Then, because $-\tilde{w}=\Delta-w \in[a+\Delta, b+\Delta]$ and $x_{1}=0,1$,

$$
\begin{aligned}
P\left(Y_{1}=1, Y_{2}=0 \mid X=x, X_{1}=x_{1}\right) & =\int F_{\eta_{1}}\left(x_{1}-w\right)\left(1-F_{\eta_{2}}(x-w)\right) f_{W}(w) d w \\
& =\int F_{\eta_{1}}\left(x_{1}-\tilde{w}\right)\left(1-F_{\tilde{\eta}_{2}}(x-\tilde{w})\right) f_{\tilde{w}}(\tilde{w}) d \tilde{w} .
\end{aligned}
$$

Similarly, because $\alpha-\tilde{w}=\alpha_{0}-w \in\left[\alpha_{0}+a, \alpha_{0}+b\right]$ and for $e \in\left(\alpha_{0}+a, \alpha_{0}+b\right] \cup\left(1+\alpha_{0}+a, 1+\alpha_{0}+b\right]$, $F_{\tilde{\eta}_{1}}(e)=F_{\eta_{1}}(e)$, we have

$$
\begin{aligned}
P\left(Y_{1}=1, Y_{2}=1 \mid X=x, X_{1}=x_{1}\right) & =\int F_{\eta_{1}}\left(x_{1}+\alpha_{0}-w\right) F_{\eta_{2}}(x-w) f_{W}(w) d w \\
& =\int F_{\eta_{1}}\left(x_{1}+\alpha-\left(w+\alpha-\alpha_{0}\right)\right) F_{\eta_{2}}(x-w) f_{W}(w) d w \\
& =\int F_{\eta_{1}}\left(x_{1}+\alpha-\tilde{w}\right) F_{\tilde{\eta_{2}}}(x-\tilde{w}) f_{\tilde{w}}(\tilde{w}) d \tilde{w}
\end{aligned}
$$

This implies $\alpha_{0}$ is not identified from the impostor $\alpha$.

\section{O Proof of Theorem K.2}

We first show that both $\sigma_{0}$ and the density of $\eta_{2}$ are identified. Note $X$ has full support. This implies the density of $V$ denoted as $f_{V}(\cdot)$ is identified via

$$
f_{V}(v)=\partial_{v} E\left(Y_{2} \mid X=v\right)
$$


In addition, we have

$$
f_{V}(\cdot)=f_{\eta_{2}} * \phi_{\sigma_{0}}(\cdot)
$$

where $*$ denotes the convolution operator. Suppose $f_{\eta_{2}}(\cdot)$ and $\sigma_{0}$ are not identified so that there exist $f_{\eta_{2}}^{\prime}(\cdot)$ and $\sigma^{\prime}$ such that

$$
f_{V}(\cdot)=f_{\eta_{2}}^{\prime} * \phi_{\sigma^{\prime}}(\cdot)
$$

Without loss of generality, we assume $\sigma^{\prime} \geq \sigma_{0}$, otherwise, we can just relabel $f_{\eta_{2}}(\cdot)$ and $f_{\eta_{2}}^{\prime}(\cdot)$. Then we have

$$
f_{\eta_{2}}(\cdot)=f_{\eta_{2}}^{\prime} * \phi_{\left(\sigma^{\prime 2}-\sigma_{0}^{2}\right)}
$$

By Assumption B5, we have $\sigma^{\prime}=\sigma_{0}$, which implies $f_{\eta_{2}}(\cdot)=f_{\eta_{2}}^{\prime}(\cdot)$.

In the following, we proceed given that $f_{\eta_{2}}(\cdot)$ and $\sigma_{0}$ are known. Recall $F_{\eta_{1}}(\cdot)$ as the CDF of $\eta_{1}$. Then,

$$
P^{11}\left(x_{1}, x\right)=P\left(Y_{1}=1, Y_{2}=1 \mid X_{1}=x_{1}, X=x\right)=\int F_{\eta_{1}}\left(x_{1}+\alpha_{0}-\gamma_{0} w\right) F_{\eta_{2}}\left(x-\sigma_{0} w\right) f_{W}(w) d w
$$

and

$$
P^{10}\left(x_{1}, x\right)=P\left(Y_{1}=1, Y_{2}=0 \mid X_{1}=x_{1}, X=x\right)=\int F_{\eta_{1}}\left(x_{1}-\gamma_{0} w\right)\left(1-F_{\eta_{2}}\left(x-\sigma_{0} w\right)\right) f_{W}(w) d w .
$$

Taking derivatives of $P^{11}\left(x_{1}, x\right)$ and $P^{10}\left(x_{1}, x\right)$ w.r.t. $x$, we have

$$
\partial_{x} P^{11}\left(x_{1}, x\right)=\int F_{\eta_{1}}\left(x_{1}+\alpha_{0}-\gamma_{0} w\right) f_{\eta_{2}}\left(x-\sigma_{0} w\right) f_{W}(w) d w
$$

and

$$
-\partial_{x} P^{10}\left(x_{1}, x\right)=\int F_{\eta_{1}}\left(x_{1}-\gamma_{0} w\right) f_{\eta_{2}}\left(x-\sigma_{0} w\right) f_{W}(w) d w .
$$

Applying Fourier transform on both sides of (O.33) and (O.34), we have

$$
\mathcal{F}\left(\partial_{x} P^{11}\left(x_{1}, \cdot\right)\right)=\mathcal{F}_{\sigma_{0}}\left(F_{\eta_{1}}\left(x_{1}+\alpha_{0}-\gamma_{0} \cdot\right) f_{W}(\cdot)\right) \mathcal{F}\left(f_{\eta_{2}}(\cdot)\right)
$$

and

$$
\mathcal{F}\left(-\partial_{x} P^{10}\left(x_{1}, \cdot\right)\right)=\mathcal{F}_{\sigma_{0}}\left(F_{\eta_{1}}\left(x_{1}-\gamma_{0} \cdot\right) f_{W}(\cdot)\right) \mathcal{F}\left(f_{\eta_{2}}(\cdot)\right),
$$


where for a generic function $g(w)$,

$$
\mathcal{F}_{\sigma_{0}}(g(\cdot))(t)=\frac{1}{\sqrt{2 \pi}} \int \exp \left(-2 \pi i t \sigma_{0} w\right) g(w) d w .
$$

Then, by $(\mathrm{O} .35)$, we can identify $F_{\eta_{1}}\left(x_{1}+\alpha_{0}-\cdot\right)$ by

$$
F_{\eta_{1}}\left(x_{1}+\alpha_{0}-\gamma_{0} \cdot\right)=\mathcal{F}_{\sigma_{0}}^{-1}\left(\frac{\mathcal{F}\left(\partial_{x} P^{11}\left(x_{1}, \cdot\right)\right)}{\mathcal{F}\left(f_{\eta_{2}}(\cdot)\right)}\right)(\cdot) / f_{W}(\cdot) .
$$

Similarly, we can identify

$$
F_{\eta_{1}}\left(x_{1}-\gamma_{0} \cdot\right)=\mathcal{F}_{\sigma_{0}}^{-1}\left(\frac{\mathcal{F}\left(-\partial_{x} P^{10}\left(x_{1}, \cdot\right)\right)}{\mathcal{F}\left(f_{\eta_{2}}(\cdot)\right)}\right)(\cdot) / f_{W}(\cdot),
$$

where for a generic function $g(w)$,

$$
\mathcal{F}_{\sigma_{0}}^{-1}(g(\cdot))(t)=\frac{\sigma_{0}}{\sqrt{2 \pi}} \int \exp \left(2 \pi i t \sigma_{0} w\right) g(w) d w
$$

By finding the two pairs $\left(\left(x_{1}, w\right),\left(x_{1}^{\prime}, w^{\prime}\right)\right)$ and $\left(\left(\tilde{x}_{1}, \tilde{w}\right),\left(\tilde{x}_{1}^{\prime}, \tilde{w}^{\prime}\right)\right)$ such that $w-w^{\prime} \neq \tilde{w}-\tilde{w}^{\prime}$,

$$
F_{\eta_{1}}\left(x_{1}+\alpha_{0}-\gamma_{0} w\right)=F_{\eta_{1}}\left(x_{1}^{\prime}-\gamma_{0} w^{\prime}\right), \quad \text { and } \quad F_{\eta_{1}}\left(\tilde{x}_{1}+\alpha_{0}-\gamma_{0} \tilde{w}\right)=F_{\eta_{1}}\left(\tilde{x}_{1}^{\prime}-\gamma_{0} \tilde{w}^{\prime}\right)
$$

we can identify both $\alpha_{0}$ and $\gamma_{0}$ as the solution of the following linear system:

$$
\alpha_{0}+\gamma_{0}\left(w^{\prime}-w\right)=x_{1}^{\prime}-x_{1} \quad \alpha_{0}+\gamma_{0}\left(\tilde{w}^{\prime}-\tilde{w}\right)=\tilde{x}_{1}^{\prime}-\tilde{x}_{1} .
$$

\section{References}

Abbring, J., And J. Heckman (2007): "Econometrics Evaluation of Social Programs, Part III:

Distributional Treatment Effects, Dynamic Treatment Effects, Dynamic Discrete Choice, and General Equilibrium Policy Evaluation," in Handbook of Econometrics, Vol. 6B, ed. by J. J. Heckman, and E. E. Leamer. North Holland.

Abowd, J. M., And D. CARD (1989): "On the Covariance Structure of Earnings and Hours Changes," Econometrica, 57(2), 411-445.

Abrevaya, J., J. Hausman, and S. Khan (2010): "Testing for Causal Effects in a Generalized Regression Model with Endogenous Regressors," Econometrica, 78(6), 2043-2061.

Ashworth, J., V. J. Hotz, A. Maurel, and T. Ransom (2020): "Changes Across Cohorts in Wage Returns to Schooling and Early Work Experiences," forthcoming, Journal of Labor Economics. 
BAI, J., And S. NG (2002): "Determining the Number of Factors in Approximate Factor Models," Econometrica, 70(1), 191-221.

BAI, J., AND S. NG (2010): "Instrumental Variable Estimation in a Data Rich Environment," Econometric Theory, 26(6), 1577-1606.

Bierens, H., And J. Hartog (1988): "Non-Linear Eegression with Discrete Explanatory Variables, with an Application to the Earnings Function," Journal of Econometrics, 38(3), 269-299.

Bonhomme, S., and J.-M. Robin (2010): "Generalized Non-Parametric Deconvolution with an Application to Earnings Dynamics," Review of Economic Studies, 77(2), 491-533.

Butucea, C., And C. Matias (2005): "Minimax estimation of the noise level and of the deconvolution density in a semiparametric convolution model," Bernoulli, 11(2), 309-340.

Carneiro, P., K. Hansen, and J. J. Heckman (2003): "Estimating Distributions of Treatment Effects with an Application to the Returns to Schooling and Measurement of the Effects of Uncertainty on College Choice," International Economic Review, 44(2), 361-422.

Carneiro, P., and S. LeE (2009): "Estimating distributions of potential outcomes using local instrumental variables with an application to changes in college enrollment and wage inequality," Journal of Econometrics, 149(2), 191-208.

Chen, S., S. Khan, and X. Tang (2016): "On the Informational Content of Special Regressors in Heteroskedastic Binary Response Models," Journal of Econometrics, 193, 162-182.

Chesher, A. (2005): "Nonparametric identification under discrete variation," Econometrica, $73(5), 1525-1550$.

Chiburis, R. (2010): "Semiparametric Bounds on Treatment Effects," Journal of Econometrics, $159(2), 267-275$.

Cunha, F., J. J. Heckman, and S. M. Schennach (2010): "Estimating the Technology of Cognitive and Noncognitive Skill Formation," Econometrica, 78(3), 883-931.

Geary, R. (1942): "Inherent relations between random variables," Proceedings of the Royal Irish Academy, 47, 63-76.

Gourieroux, C., A. Monfort, and J.-P. Renne (2017): "Statistical inference for independent component analysis: Application to structural VAR models," Journal of Econometrics, 196(1), 111-126.

HAN, A. (1987): "Non-parametric analysis of a generalized regression model: The maximum rank correlation estimator," Journal of Econometrics, 35(2-3), 303-316. 
Han, S., And S. LeE (2019): "Estimation in a Generalization of a Bivariate Probit Models with Dummy Endogenous Regressors," Journal of Applied Econometrics, 34(6), 994-1015.

HAN, S., AND E. J. Vytlacil (2017): "Identification in a generalization of bivariate probit models with endogenous regressors," Journal of Econometrics, 199(1), 63-73.

Heckman, J., and S. Navarro (2007): "Dynamic Discrete Choice and Dynamic Treatment Effects," Journal of Econometrics, 136(2), 341-396.

Heckman, J. J., J. Humphries, and G. Veramendi (2018): "Returns to Education: The Causal Effects of Education on Earnings, Health, and Smoking," Journal of Political Economy, 126, S197-S246.

Heckman, J. J., And E. Vytlacil (2005): "Structural equations, treatment effects, and econometric policy evaluation 1," Econometrica, 73(3), 669-738.

Heckman, J. J., And E. J. Vytlacil (2007a): "Econometric evaluation of social programs, part I: Causal models, structural models and econometric policy evaluation," Handbook of econometrics, 6, 4779-4874.

(2007b): "Econometric evaluation of social programs, part II: Using the marginal treatment effect to organize alternative econometric estimators to evaluate social programs, and to forecast their effects in new environments," Handbook of econometrics, 6, 4875-5143.

Henderson, D., Q. Li, C. Parmeter, and S. Yao (2015): "Gradient-based Smoothing Parameter Selection for Nonparametric Regression Estimation," Journal of Econometrics, 184, 233-241.

Horowitz, J. (1992): "A Smoothed Maximum Score Estimator for the Binary Response Model," Econometrica, 60(3).

Hu, Y., And S. M. Schennach (2008): "Instrumental variable treatment of nonclassical measurement error models," Econometrica, 76(1), 195-216.

Hu, Y., And S. M. Schennach (2013): "Nonparametric identification and semiparametric estimation of classical measurement error models without side information," Journal of the American Statistical Association, 108(501), 177-186.

Hyvärinen, A., AND E. OJA (2000): Independent component analysis: algorithms and applications, vol. 13. Elsevier.

Jochmans, K. (2013): "Pairwise-comparison estimation with nonparametric controls," Econometrics Journal, 16, 340-372.

Khan, S. (2001): "Two Stage Rank Estimation of Quantile Index Models," Journal of Econometrics, 100, 319-355. 
Khan, S., And D. NeKIPElov (2018): "Information structure and statistical information in discrete response models," Quantitative Economics, 9(2), 995-1017.

Khan, S., And E. TAmer (2018): "Discussion of "Simple Estimators for Invertible Index Models" by Ahn et al.," Journal of Business \& Economic Statistics, 36, 11-15.

Klein, R., C. Shan, and F. Vella (2015): "Estimation of marginal effects in semiparametric selection models with binary outcomes," Journal of Econometrics, 185(1), 82-94.

Klein, R., and R. Spady (1993): "An Efficient Semiparametric Estimator for Binary Response Model," Econometrica, 61(2), 387-421.

Lewbel, A. (2000): "Semiparametric Qualitative Response Model Estimation with Unknown Heteroscedasticity or Instrumental Variables," Journal of Econometrics, 97(1), 145-177.

Lewbel, A., S. M. Schennach, and L. Zhang (2020): "Identification of a Triangular Two Equation System Without Instruments," Working Paper.

Moneta, A., D. E. P. O. Hoyer, and A. CoAd (2013): "Causal inference by independent component analysis: Theory and applications," Oxford Bulletin of Economics and Statistics, $75(5), 705-730$.

MourifiÉ, I. (2015): "Sharp Bounds on Treatment Effects in a Binary Triangular System," Journal of Econometrics, 187(1), 74-81.

Newey, W., and D. McFadden (1994): "Large Sample Estimation and Hypothesis Testing," in Handbook of Econometrics, Vol. 4, ed. by R. Engle, and D. McFadden. North Holland.

Powell, J., J. Stock, and T. Stoker (1989): "Semiparametric Estimation of Index Coefficients," Econometrica, pp. 1403-1430.

Reiersol, O. (1950): "Identifiability of a Linear Relation Between Variables Which are Subject to Error," Econometrica, 18(4), 375-389.

Shaikh, A. M., and E. Vytlacil (2011): "Partial Identification in Triangular Systems of Equations with Binary Dependent Variables," Econometrica, 79(3), 949-955.

Sherman, R. (1993): "The Limiting Distribution of the Maximum Rank Correlation Estimator," Econometrica, 61, 123-137.

(1994a): "Maximal Inequalities for Degenerate U-Processes with Applications to Optimization Estimators," Annals of Statistics, 22, 439-459.

(1994b): "U-Processes in the Analysis of a Generalized Semiparametric Regression Estimator," Econometric Theory, 10, 372-395. 
TAmer, E. (2003): "Incomplete Bivariate Discrete Response Model with Multiple Equilibria," Review of Economic Studies, 70(1), 147-167.

Vuong, Q., AND H. Xu (2017): "Counterfactual mapping and individual treatment effects in nonseparable models with binary endogeneity," Quantitative Economics, 8(2), 589-610.

Vytlacil, E. J., and N. Yildiz (2007): "Dummy Endogenous Variables in Weakly Separable Models," Econometrica, 75(3), 757-779. 